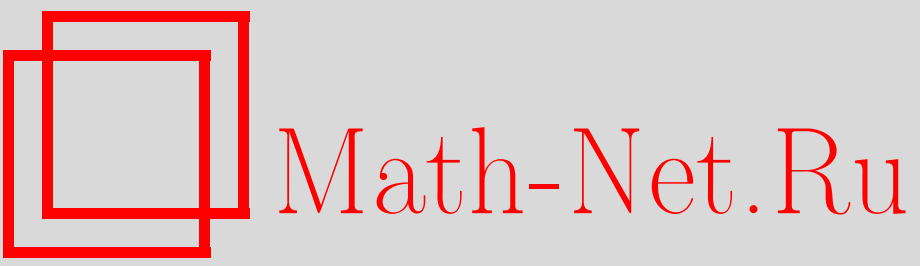

П. И. Пронин, К. В. Степаньянц, Однопетлевые расходимости в теориях с произвольным неминимальным оператором в искривленном пространстве, $T M \Phi$, 1997, том 110, номер 3, 351-371

DOI: https://doi.org/10.4213/tmf974

Использование Общероссийского математического портала Math-Net.Ru подразумевает, что вы прочитали и согласны с пользовательским соглашением

http://www.mathnet.ru/rus/agreement

Параметры загрузки:

IP : 52.87 .193 .239

26 апреля 2023 г., 14:50:11 


\section{ОДНОПЕТЛЕВЫЕ РАСХОДИМОСТИ В ТЕОРИЯХ \\ С ПРОИЗВОЛЬНЫМ НЕМИНИМАЛЬНЫМ ОПЕРАТОРОМ В ИСКРИВЛЕННОМ ПРОСТРАНСТВЕ}

Построена явная формула для расходящейся части однопетлевого эффективного действия для произвольного неминимального оператора в четырехмерном искривленном пространстве.

\section{ВВЕДЕНИЕ}

Вычисление расходяшейся части однопетлевого эффективного действия [1]

$$
\Gamma[\varphi]=S[\varphi]+\frac{i}{2} \hbar \operatorname{tr} \ln D+O\left(\hbar^{2}\right)
$$

где

$$
D_{i}{ }^{j} \equiv \frac{\delta^{2} S}{\delta \varphi^{i} \delta \varphi_{j}},
$$

по-прежнему остается одной из наиболее часто встречающихся операций при исследовании моделей теории калибровочных полей и квантовой гравитации. В настоящее время для ее реализации существует целый ряд методов, наиболее известными из которых являются метод собственного времени Швингера-де Витта [2, 3] и фейнмановская диаграммная техника.

K сожалению, в искривленном пространстве стандартный диаграммный метод не является явно ковариантным, и его применение весьма затруднительно. Явная ковариантность, а также достаточно тривиальная реформулировка в геометрических терминах являются несомненными достоинствами метода Швингера-де Витта. Однако с его помощью оказалось возможным найти алгоритм вычисления расходящейся части однопетлевого эффективного действия только для простейшего минимального оператора второго порядка.

В работе [4] показано, что метод Швингера-де Витта позволяет явно ковариантным образом вычислять фейнмановские диаграммы, в которых пропагатор зависит от фоновой метрики. Поскольку эффективное действие есть сумма вкладов одночастично неприводимых диаграмм, то при помоши данного обобщения метода Швингера-де Витта

\footnotetext{
* Физический факультет Московского государственного университета pronin@theor.phys.msu.su, stepan@theor.phys.msu.su
} 
оказывается в принципе возможным строить алгоритмы для произвольных дифференциальных операторов. В частности, в [4] получены явные выражения для расходящейся части однопетлевого эффективного действия в теориях с простейшими операторами второго и четвертого порядков.

Однако диаграммный метод, обобшенный на технику фонового поля в работе [5], также может быть успешно применен для проведения вычислений. В частности, в рамках данного подхода в работах $[6,7]$ были вычислены однопетлевые контрчлены для произвольного минимального оператора вида

$$
\begin{aligned}
& D_{i}{ }^{j}=\delta_{i}{ }^{j} \square^{l}+S^{\mu_{1} \mu_{2} \ldots \mu_{2 l-1}}{ }_{i}{ }^{j} \nabla_{\mu_{1}} \nabla_{\mu_{2}} \ldots \nabla_{\mu_{2 l-1}}+ \\
& +W^{\mu_{1} \mu_{2} \ldots \mu_{2 l-2}{ }_{i}^{j}} \nabla_{\mu_{1}} \nabla_{\mu_{2}} \ldots \nabla_{\mu_{2 l-2}}+
\end{aligned}
$$

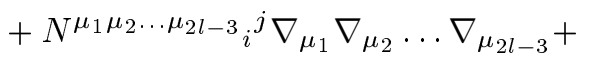

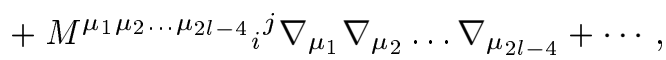

где $\square \equiv \nabla_{\mu} \nabla^{\mu}$, а $\nabla_{\mu}-$ ковариантная производная:

$$
\begin{aligned}
\nabla_{\alpha} T^{\beta}{ }_{i}{ }^{j} & =\partial_{\alpha} T^{\beta}{ }_{i}{ }^{j}+\Gamma_{\alpha \gamma}^{\beta} T_{i}^{\gamma}{ }^{j}+\omega_{\alpha i}{ }^{k} T_{k}^{\beta}{ }_{k}^{j}-T_{i}^{\beta}{ }_{i} \omega_{\alpha k}{ }^{j} \\
\nabla_{\mu} \Phi_{i} & =\partial_{\mu} \Phi_{i}+\omega_{\mu i}{ }^{j} \Phi_{j} .
\end{aligned}
$$

Детальное изложение метода расчета приведено в работе [7].

Цель данной работы, являющейся ее органическим продолжением, - построение явного выражения для однопетлевого эффективного действия в теории с произвольным дифференциальным оператором вида

$$
\begin{aligned}
& D_{i}{ }^{j}=K^{\mu_{1} \mu_{2} \ldots \mu_{L}}{ }_{i}^{j} \nabla_{\mu_{1}} \nabla_{\mu_{2}} \ldots \nabla_{\mu_{L}}+S^{\mu_{1} \mu_{2} \ldots \mu_{L-1}}{ }_{i}^{j} \nabla_{\mu_{1}} \nabla_{\mu_{2}} \ldots \nabla_{\mu_{L-1}}+
\end{aligned}
$$

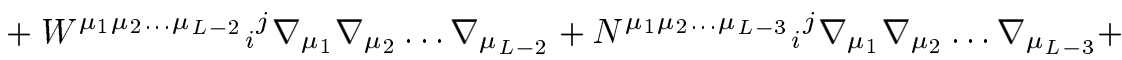

$$
\begin{aligned}
& +M^{\mu_{1} \mu_{2} \ldots \mu_{L-4}{ }_{i}^{j}} \nabla_{\mu_{1}} \nabla_{\mu_{2}} \ldots \nabla_{\mu_{L-4}}+\cdots
\end{aligned}
$$

без каких-либо ограничений на его форму и порядок на фоне искривленного пространства-времени (случай плоского пространства был исследован в [6]). Поля $K, S, W$, $N, M, \ldots$ мы будем считать симметричными по греческим индексам. Выполнения данного условия всегда можно добиться при помощи коммутации ковариантных производных.

В случае $L=2 l$ и $K^{\mu \nu \ldots \alpha}{ }_{i}{ }^{j}=K_{0}{ }^{\mu \nu \ldots \alpha} \delta_{i}{ }^{j}$, где $K_{0}$ - полностью симметричный тензор, построенный из $g_{\mu \nu}$,

$$
K_{0}{ }^{\mu \nu \alpha \beta \cdots}=\frac{1}{(2 l-1) ! !}\left(g_{\mu \nu} g_{\alpha \beta} \cdots+g_{\mu \alpha} g_{\nu \beta} \cdots+\cdots\right)
$$

оператор (4) совпадает с (2) с точностью до перестановок ковариантных производных. Расходящаяся часть однопетлевого эффективного действия в данном случае также была найдена в [6]. 


\section{2. ДИАГРАММНАЯ ТЕХНИКА В МЕТОДЕ ФОНОВОГО ПОЛЯ}

Для теории с действием $S[\varphi]$ эффективное действие имеет вид (1) и представляет собой некоторый дифференциальньй оператор, зависяший от "фонового" поля $\varphi$ (латинские буквы обозначают весь набор индексов поля $\varphi$ ).

Первое слагаемое в (1) соответствует древесному приближению, а второе является суммарным однопетлевым вкладом.

Вычисление расходящейся части (1) может быть выполнено при помоши фейнмановской диаграммной техники. Для этого член с наибольшим количеством производных необходимо записать в виде

$$
K^{\mu_{1} \ldots \mu_{L}{ }_{i}{ }^{j}}(\eta) \partial_{\mu_{1}} \ldots \partial_{\mu_{L}}+O(h, \omega, \phi) \equiv(K \partial)_{i}{ }^{j}+O(h, \omega, \phi),
$$

где $g_{\mu \nu}=\eta_{\mu \nu}+h_{\mu \nu}$ и предполагается, что $K$ зависит только от метрического тензора. Заметим, что в данном выражении имеются некоторые дополнительные поля $\phi$. Далее мы подробно рассмотрим причины их появления.

Теперь выражение для однопетлевого эффективного действия (1) можно переписать следуюшим образом:

$$
\begin{aligned}
\Gamma^{(1)}= & \frac{i}{2} \operatorname{tr} \ln D_{i}{ }^{j}=\frac{i}{2} \operatorname{tr} \ln ((K \partial)+V)=\frac{i}{2} \operatorname{tr} \ln (K \partial)+ \\
& +\frac{i}{2} \operatorname{tr} \ln \left(1+(K \partial)^{-1} V\right)=\frac{i}{2} \operatorname{tr} \ln (K \partial)+\frac{i}{2} \operatorname{tr} \sum_{k=1}^{\infty} \frac{1}{k}\left(-\frac{1}{(K \partial)} V\right)^{k},
\end{aligned}
$$

где

$$
\begin{aligned}
V \equiv & S^{\mu_{1} \ldots \mu_{L-1}} \partial_{\mu_{1}} \ldots \partial_{\mu_{L-1}}+W^{\mu_{1} \ldots \mu_{L-2}} \partial_{\mu_{1}} \ldots \partial_{\mu_{L-2}}+N^{\mu_{1} \ldots \mu_{L-3}} \partial_{\mu_{1}} \ldots \partial_{\mu_{L-3}}+ \\
& +M^{\mu_{1} \ldots \mu_{L-4}} \partial_{\mu_{1}} \ldots \partial_{\mu_{L-4}}+\cdots+O(h, \omega, \phi)
\end{aligned}
$$

Первое слагаемое в формуле (7) является некоторой численной постоянной (бесконечной), которая может быть отброшена. Второе слагаемоеграфически представляется как сумма однопетлевых диаграмм с $k$ вершинами. Пропагатор в импульсном представлении, очевидно, имеет вид $(K k)^{-1}{ }_{i}^{j}$, где

$$
(K k)_{i}{ }^{j} \equiv K^{\mu \nu \ldots \alpha}{ }_{i}{ }^{j} k_{\mu} k_{\nu} \ldots k_{\alpha} ; \quad(K k)^{-1}{ }_{i}{ }^{m}(K k)_{m}{ }^{j}=\delta_{i}{ }^{j} .
$$

Далее мы всегда будем предполагать, что $(K k)^{-1}{ }_{i}{ }^{j}$ сушествует, что обычно достигается при помоши фиксации калибровки для всех имеющихся инвариантностей.

В случае плоского пространства расходятся только диаграммы, представленные на рис. 1. Их вычисление производится с использованием размерной регуляризации. В [6] было показано, что для получения расходящейся части размерно регуляризованного интеграла необходимо удержать только логарифмически расходящиеся члены, а затем выполнить интегрирование согласно следуюшим соотношениям:

$$
\begin{aligned}
\int d^{d} k \frac{1}{k^{2 m+5}} k_{\mu_{1}} k_{\mu_{2}} \ldots k_{\mu_{2 m+1}} & =0 \\
\int d^{d} k \frac{1}{k^{2 m+4}} k_{\mu_{1}} k_{\mu_{2}} \ldots k_{\mu_{2 l}} & =-\frac{2 i \pi^{2}}{d-4}\left\langle n_{\mu_{1}} n_{\mu_{2}} \ldots n_{\mu_{2 m}}\right\rangle
\end{aligned}
$$




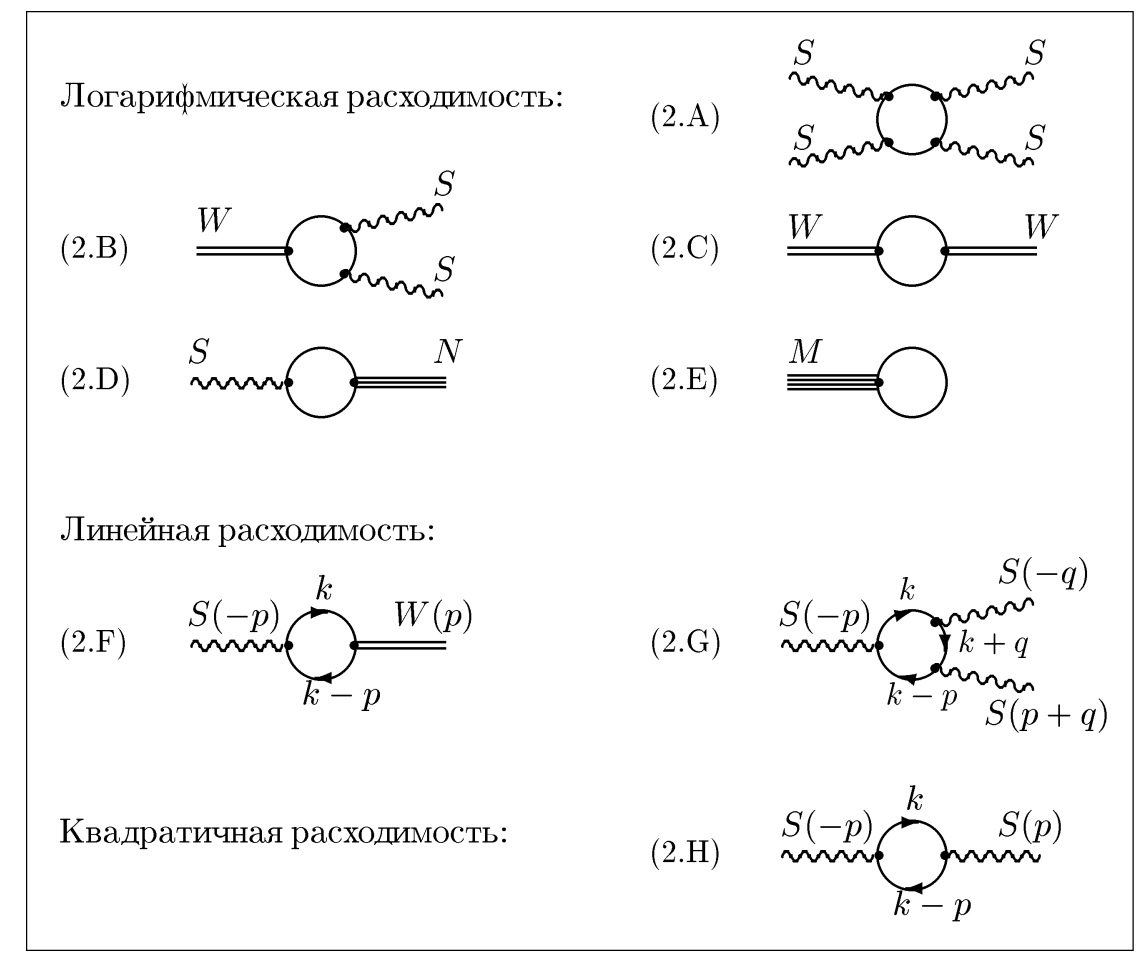

Рис. 1. Расходящиеся диаграммы в плоском пространстве.

где $n_{\mu}$ - единичный вектор $\left(n_{\mu} n^{\mu}=1\right)$, а

$$
\begin{aligned}
\left\langle n_{\mu_{1}} n_{\mu_{2}} \ldots n_{\mu_{2 m}}\right\rangle= & \frac{1}{2^{m}(m+1) !}\left(g_{\mu_{1} \mu_{2}} g_{\mu_{3} \mu_{4}} \ldots g_{\mu_{2 m-1} \mu_{2 m}}+\right. \\
& \left.+g_{\mu_{1} \mu_{3}} g_{\mu_{2} \mu_{4}} \ldots g_{\mu_{2 m-1} \mu_{2 m}}+\ldots\right)
\end{aligned}
$$

- результат интегрирования по угловым переменным (суммирование в (11) производится по всевозможным способам расстановки индексов).

Суммарный вклад диаграмм, приведенных на рис. 1, был вычислен в [6] и имеет вид

$$
\begin{aligned}
\left(\Gamma_{\infty}^{(1)}\right)^{\text {flat }}= & \frac{1}{16 \pi^{2}(d-4)} \operatorname{tr} \int d^{4} x\left\langle\frac{1}{4} \widehat{S}^{4}-\widehat{W} \widehat{S}^{2}+\frac{1}{2} \widehat{W}^{2}+\widehat{S} \widehat{N}-\widehat{M}-L \partial_{\mu} \widehat{S} \widehat{W} \widehat{K}^{\mu}+\right. \\
& +(L-2) \partial_{\mu} \widehat{S} \widehat{W}^{\mu}+\frac{1}{2} L(L-1) \partial_{\mu} \widehat{S} \partial_{\nu} \widehat{S}^{\nu} \widehat{K}^{\mu}-\frac{1}{4}(L-1)(L-2) \partial_{\mu} \widehat{S} \partial_{\nu} \widehat{S}^{\mu \nu}- \\
& -\frac{1}{2} \partial_{\mu} \widehat{S} \partial_{\nu} \widehat{S}\left(-\frac{1}{2} L(L-1) \widehat{K}^{\mu \nu}+L^{2} \widehat{K}^{\mu} \widehat{K}^{\nu}\right)+\frac{1}{3}\left((L-1) \partial_{\mu} \widehat{S}^{\mu} \widehat{S}^{2}-\right. \\
& \left.\left.-L \partial_{\mu} \widehat{S} \widehat{K}^{\mu} \widehat{S}^{2}-(L-1) \partial_{\mu} \widehat{S} \widehat{S} \widehat{S}^{\mu}+L \partial_{\mu} \widehat{S} \widehat{S}^{2} \widehat{K}^{\mu}\right)\right\rangle,
\end{aligned}
$$

где были введены следуюшие обозначения:

$$
\begin{gathered}
(K n) \equiv K^{\mu \nu \ldots \beta} n_{\mu} n_{\nu} \ldots n_{\beta}, \quad(K n)^{-1}{ }_{i}^{m}(K n)_{m}{ }^{j}=\delta_{i}{ }^{j}, \\
\widehat{W} \equiv(K n)^{-1} W^{\mu \nu \ldots \alpha} n_{\mu} n_{\nu} \ldots n_{\alpha}, \quad \widehat{K}^{\alpha} \equiv(K n)^{-1} K^{\mu \nu \ldots \beta \alpha} n_{\mu} n_{\nu} \ldots n_{\beta}
\end{gathered}
$$


(3.A)

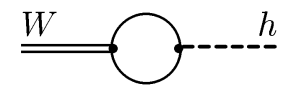

$S$
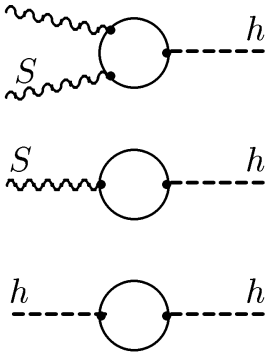

$S$
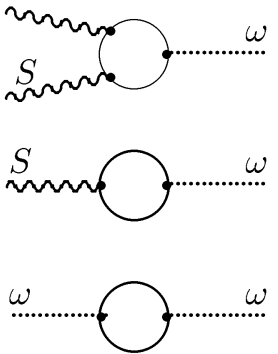

$(2 . \mathrm{O})$

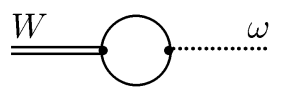

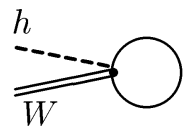

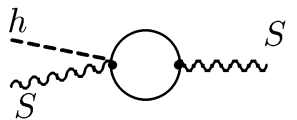

$h$<smiles>C1CCC2(CC1)CCCC2</smiles><smiles>CC1([Te])CCCCC1</smiles>

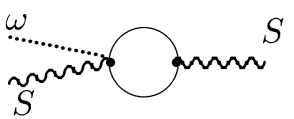<smiles>[As]C1([As])CCCCC1</smiles><smiles>[C]1CCCCC1</smiles>

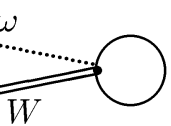

Рис. 2. Диаграммы для вычисления эффективного действия в искривленном пространстве.

и т.д.

Произведем обобшение полученных результатов на случай риманова многообразия. При этом мы немедленно сталкиваемся с тем, что число расходяшихся диаграмм в искривленном пространстве бесконечно, так как вершины, содержашие $h_{\mu \nu}$, имеют степень импульса $L$ (а следовательно, индекс расходимости не понижается с увеличением числа вершин) и, кроме того, число типов вершин также бесконечно.

Однако окончательное выражение должно быть инвариантно относительно общекоординатных преобразований. Это позволяет получить результат при помощи вычисления только некоторого конечного числа расходяшихся диаграмм. Очевидно, мы должны произвести замену обычных производных в (12) на ковариантные и, как и в случае минимального оператора, добавить слагаемые, содержащие тензоры кривизны

$$
\begin{aligned}
R_{\beta \mu \nu}^{\alpha} & =\partial_{\mu} \Gamma_{\nu \beta}^{\alpha}-\partial_{\nu} \Gamma_{\mu \beta}^{\alpha}+\Gamma_{\mu \gamma}^{\alpha} \Gamma_{\nu \beta}^{\gamma}-\Gamma_{\nu \gamma}^{\alpha} \Gamma_{\mu \beta}^{\gamma}, \\
F_{\mu \nu i}{ }^{j} & =\partial_{\mu} \omega_{\nu i}{ }^{j}-\partial_{\nu} \omega_{\mu i}{ }^{j}+\omega_{\mu i}{ }^{k} \omega_{\nu k}{ }^{j}-\omega_{\nu i}{ }^{k} \omega_{\mu k}{ }^{j} .
\end{aligned}
$$

Как и в случае минимального оператора, вид дополнительных слагаемых может быть определен при помоши вычисления фейнмановских диаграмм в приближении слабого 
Квадратичная расходимость:

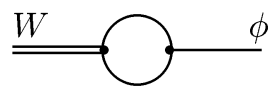

Расходимость третьего порядка:

Расходимость четвертого порядка:
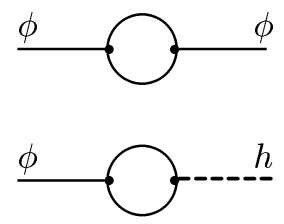

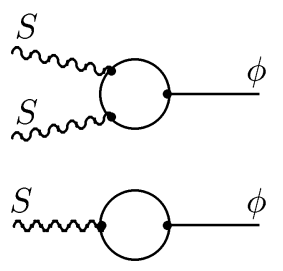

$(3 . G)$

Рис. 3. Диаграммы для неминимального оператора, соответствующие разложению по слабому полю $\partial_{\alpha} \phi^{b}$ в первом неисчезающем приближении.

поля. Однако теперь здесь имеется ряд существенных отличий. Одно из них состоит в том, что нам неизвестна зависимость $K=K\left(g_{\mu \nu}\right)$, а следовательно, и выражения для пропагатора и вершин в приближении слабого поля, получаюшихся при разложении оператора по $h_{\mu \nu}$.

Чтобы обойти данную трудность, будем использовать следуюший прием. Предполо-

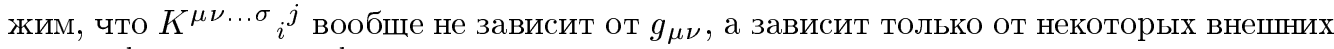
полей $\phi^{b}$, причем $\partial_{\alpha} \phi^{b}$ будем считать слабым полем. Однако будем налагать условие

$$
\begin{aligned}
\nabla_{\alpha} K^{\mu \nu \ldots \sigma}{ }_{i}^{j}= & \frac{\partial K^{\mu \nu \ldots \sigma}}{\partial \phi^{b}} \partial_{\alpha} \phi^{b}+\Gamma_{\alpha \beta}^{\mu} K^{\beta \nu \ldots \sigma}+\cdots+\Gamma_{\alpha \beta}^{\sigma} K^{\mu \nu \ldots \beta}+ \\
& +\omega_{\alpha i}{ }^{k} K^{\mu \nu \ldots \sigma}{ }_{k}{ }^{j}-K^{\mu \nu \ldots \sigma_{i}{ }^{k} \omega_{\alpha k}{ }^{j}}=0
\end{aligned}
$$

которое, очевидно, вьполнено, если $K^{\mu \nu \ldots \sigma}{ }_{i}{ }^{j}$ зависит только от $g_{\mu \nu}$.

Условие (15) может быть выполнено только в том случае, если поле $\omega_{\alpha i}{ }^{j}$ рассматривается как слабое. Это является вполне естественным, если вспомнить, что, как правило, оно строится из символов Кристоффеля $\Gamma_{\mu \nu}^{\sigma}$. Таким образом, слагаемые, содержашие связность, необходимо вычислять также при помощи разложения по слабым полям (в случае минимального оператора это не является обязательным).

Кроме того, помимо диаграмм с внешними $h$ - и $\omega$-линиями, аналогичных случаю минимального оператора (см. рис. 2), теперь, в случае неминимального оператора, необходимо добавить ряд диаграмм, содержаших внешние $\phi$-линии. Они приведены на рис. 3.

Несложно видеть (см. также раздел 3 ), что при вычислении данных диаграмм появляются слагаемые, содержашие $\partial_{\alpha} K^{\mu \nu \ldots \sigma}{ }_{i}^{j}$. Выражая их при помоши (15) через метрику и связность, мы получим окончательньй результат. 
Вычисление диаграмм, приведенных на рис. 2, показывает, что ответы как для метрики, так и для связности невозможно представить в виде предела слабого поля некоторого ковариантного выражения. Однако после добавления диаграмм, содержаших внешние $\phi$-линии, ковариантность восстанавливается.

Таким образом, в случае неминимального оператора уже невозможно раздельно вычислять члены, содержашие тензоры кривизны, построенные из метрики $\left(R_{\beta \mu \nu}^{\alpha}\right)$ и связности $\left(F_{\mu \nu}\right)$.

Данные рассуждения будут проиллюстрированы в разделе 3 на примере вычисления простейшей группы диаграмм.

\section{3. НЕМИНИМАЛЬНЫЙ ОПЕРАТОР НА ФОНЕ ИСКРИВЛЕННОГО ПРОСТРАНСТВА}

Перейдем теперь к конкретному вычислению расходящихся диаграмм, указанных в предыдущем разделе. В случае неминимального оператора они являются чрезвычайно громоздкими, поэтому мы подробно рассмотрим только одну, простейшую группу диаграмм, приводящих к членам $W R$ и $W F$.

В предположении, что $K^{\mu \nu \ldots \alpha}$ не зависит от метрики, вершины взаимодействия, содержащие $h_{\mu \nu}$, получаются при помощи разложения ковариантных производных в операторе (4) до первого порядка. Затем мы производим разложение по внешним импульсам, сохраняя только логарифмически расходящиеся слагаемые. Производя интегрирование согласно предписаниям в разделе 2, находим окончательное выражение для диаграммы.

Для квадратичной диаграммы (2.А) при помощи данного метода мы легко получаем, что ее расходящаяся часть может быть записана в виде

$$
\begin{aligned}
(2 . \mathrm{A})_{\infty}= & \frac{1}{16 \pi^{2}(d-4)} \operatorname{tr}\left\langle\frac{1}{3} L(L-1)(L-2) \widehat{W} \widehat{K}^{\mu \nu \alpha} p_{\alpha} \Gamma_{\mu \nu}^{\sigma} n_{\sigma}+\right. \\
& \left.+\frac{1}{2} L(L-1) \widehat{W} \widehat{K}^{\mu \nu} \Gamma_{\mu \nu}^{\sigma} p_{\sigma}+\frac{1}{2} L(L-1) \widehat{W} \widehat{K}^{\mu \nu} \Delta^{\alpha} \Gamma_{\mu \nu}^{\sigma} n_{\sigma} p_{\alpha}\right\rangle
\end{aligned}
$$

где $\Delta^{\mu}$ есть первый коэффициент в разложении пропагатора. Его явное выражение получено в приложении.

При помощи несложных вычислений аналогичным образом находим

$$
(2 . \mathrm{B})_{\infty}=\frac{1}{6}(L-2)(L-3)(L-4) \widehat{W}^{\mu \alpha \beta} p_{\mu} \Gamma_{\alpha \beta}^{\sigma} n_{\sigma}
$$

Используя правила, указанные в приложении, последнее выражение можно переписать следующим образом:

$$
\begin{aligned}
& \frac{1}{16 \pi^{2}(d-4)} \operatorname{tr}\left\langle\frac { 1 } { 3 } ( p _ { \alpha } \Gamma _ { \mu \nu } ^ { \sigma } + p _ { \mu } \Gamma _ { \nu \alpha } ^ { \sigma } + p _ { \nu } \Gamma _ { \mu \alpha } ^ { \sigma } ) \widehat { W } n _ { \sigma } \left(\frac{1}{6} L(L-1)(L-2) \widehat{K}^{\mu \nu \alpha}+\right.\right. \\
& \left.\quad+\frac{1}{2} L(L-1) \widehat{K}^{\mu \nu} \Delta^{\alpha}+L \widehat{K}^{\alpha} \Delta^{\mu \nu}\right)+ \\
& \left.\quad+\frac{1}{3}\left(p_{\sigma} \Gamma_{\mu \nu}^{\sigma}+2 p_{\mu} \Gamma_{\nu \sigma}^{\sigma}\right) \widehat{W}\left(\frac{1}{2} L(L-1) \widehat{K}_{\mu \nu}+L \widehat{K}^{\mu} \Delta^{\nu}\right)\right\rangle .
\end{aligned}
$$


Складывая выражения для двух диаграмм, приходим к следующему результату:

$$
\begin{aligned}
& \frac{1}{16 \pi^{2}(d-4)} \operatorname{tr}\left\langle\frac{1}{2} L(L-1)(L-2) \widehat{W} \widehat{K}^{\mu \nu \alpha} p_{\alpha} \Gamma_{\mu \nu}^{\sigma} n_{\sigma}+\frac{1}{3} L(L-1) \widehat{W} \widehat{K}^{\mu \nu} \times\right. \\
& \quad \times \Delta^{\alpha} n_{\sigma}\left(2 p_{\alpha} \Gamma_{\mu \nu}^{\sigma}+p_{\mu} \Gamma_{\nu \alpha}^{\sigma}\right)+\frac{1}{3} L \widehat{W} \widehat{K}^{\alpha} \Delta^{\mu \nu} n_{\sigma}\left(p_{\alpha} \Gamma_{\mu \nu}^{\sigma}+2 p_{\mu} \Gamma_{\nu \alpha}^{\sigma}\right)+ \\
& \left.\quad+\frac{1}{3} L(L-1) \widehat{W} \widehat{K}^{\mu \nu}\left(2 p_{\sigma} \Gamma_{\mu \nu}^{\sigma}+p_{\mu} \Gamma_{\nu \sigma}^{\sigma}\right)+\frac{1}{3} L \widehat{W}^{\mu} \widehat{K}^{\nu}\left(p_{\sigma} \Gamma_{\mu \nu}^{\sigma}+2 p_{\mu} \Gamma_{\nu \sigma}^{\sigma}\right)\right\rangle .
\end{aligned}
$$

Необходимо также произвести учет связности $\omega_{\alpha i}{ }^{j}$. Ранее было замечено, что связность $\omega_{\alpha i}^{j}$ в случае неминимального оператора должна рассматриваться как слабое поле. Поэтому можно вычислить диаграммы, соответствуюшие первому неисчезаюшему приближению в разложении по $\omega_{\alpha i}{ }^{j}$, а затем по ковариантности восстановить полный результат.

В данном случае необходимо рассмотреть две линейно расходящиеся диаграммы (2.O) и (2.P). Выделяя логарифмически расходящиеся члены, легко находим

$$
\begin{aligned}
& (2 . \mathrm{O})_{\infty}=\frac{1}{16 \pi^{2}(d-4)} \operatorname{tr}\left\langle-\frac{1}{2} L(L-1) \widehat{W} \widehat{K}^{\mu \nu} \omega_{\mu} p_{\nu}-L \widehat{W} \widehat{K}^{\mu} \omega_{\mu} \Delta^{\nu} p_{\nu}\right\rangle, \\
& (2 . \mathrm{P})_{\infty}=-\frac{1}{16 \pi^{2}(d-4)} \operatorname{tr}\left\langle L(L-1) \widehat{W}^{\mu \nu} \omega_{\mu} p_{\nu}\right\rangle-\frac{1}{16 \pi^{2}(d-4)} \operatorname{tr}\left\langle\widehat{W} p_{\nu} \omega_{\mu} \Delta^{\mu \nu}\right\rangle .
\end{aligned}
$$

Суммарный вклад двух диаграмм тогда принимает вид

$$
\frac{1}{16 \pi^{2}(d-4)} \operatorname{tr}\left\langle-\frac{1}{2} L(L-1) \widehat{W} \widehat{K}^{\mu \nu} p_{\nu} \omega_{\mu}-\widehat{W} p_{\nu} \omega_{\mu} \Delta^{\mu \nu}-L \widehat{W} \widehat{K}^{\mu} p_{\nu} \omega_{\mu} \Delta^{\nu}\right\rangle .
$$

Как уже отмечалось ранее, выражения (19) и (21) не могут быть записаны в ковариантном виде. Для этого нужно учесть вклад полей $\phi^{b}$, что достигается вычислением квадратично расходящейся диаграммы (3.А), выражение для которой имеет вид

$$
(3 . \mathrm{A})=\frac{1}{16 \pi^{2}(d-4)} \operatorname{tr} \int d^{d} k(W k)(K k)^{-1} \frac{\partial(K(k-p))}{\partial \phi^{b}} \phi^{b}(K(k-p))^{-1} .
$$

После разложения до второго порядка по внешнему импульсу $p$ с сохранением только квадратичных членов (при этом выделяются логарифмически расходяшиеся слагаемые) получаем

$$
\begin{aligned}
(3 . \mathrm{A})_{\infty}= & \frac{1}{16 \pi^{2}(d-4)} \operatorname{tr}\left\langle\frac{1}{2} L(L-1) \widehat{W}(K n)^{-1} \frac{\partial(K n)^{\mu \nu}}{\partial \phi^{b}} \phi^{b} p_{\mu} p_{\nu}+\right. \\
& \left.+L \widehat{W}(K n)^{-1} \frac{\partial(K n)^{\mu}}{\partial \phi^{b}} \phi^{b} \Delta^{\nu} p_{\mu} p_{\nu}+\widehat{W}(K n)^{-1} \frac{\partial(K n)}{\partial \phi^{b}} \phi^{b} \Delta^{\mu \nu} p_{\mu} p_{\nu}\right\rangle
\end{aligned}
$$

что может быть записано в виде

$$
\begin{aligned}
& \frac{1}{16 \pi^{2}(d-4)} \operatorname{tr}\left\langle\frac{1}{2} L(L-1) \widehat{W}(K n)^{-1} \partial_{\nu} \partial_{\mu}(K n)^{\mu \nu}+\right. \\
& \left.\quad+L \widehat{W}(K n)^{-1} \partial_{\nu} \partial_{\mu}(K n)^{\mu} \Delta^{\nu}+\widehat{W}(K n)^{-1} \partial_{\nu} \partial_{\mu}(K n) \Delta^{\mu \nu}\right\rangle .
\end{aligned}
$$


Согласно условию (15) данное выражение может быть переписано следуюшим образом:

$$
\begin{aligned}
(3 . \mathrm{A})_{\infty}= & -\frac{1}{16 \pi^{2}(d-4)} \operatorname{tr}\left\langle\frac { 1 } { 2 } L ( L - 1 ) p _ { \nu } \widehat { W } \left((L-2) \Gamma_{\mu \alpha}^{\sigma} \widehat{K}^{\mu \nu \alpha} n_{\sigma}+\right.\right. \\
& \left.+\Gamma_{\mu \alpha}^{\mu} \widehat{K}^{\nu \alpha}+\Gamma_{\mu \alpha}^{\nu} \widehat{K}^{\mu \alpha}+(K n)^{-1} \omega_{\mu}(K n)^{\mu \nu}-\widehat{K}^{\mu \nu} \omega_{\mu}\right)+ \\
& +L p_{\nu} \widehat{W}\left((L-1) \Gamma_{\mu \alpha}^{\sigma} \widehat{K}^{\mu \alpha} n_{\sigma}+\Gamma_{\mu \alpha}^{\mu} \widehat{K}^{\alpha}+(K n)^{-1} \omega_{\mu}(K n)^{\mu}-\widehat{K}^{\mu} \omega_{\mu}\right) \Delta^{\nu}+ \\
& \left.+p_{\nu} \widehat{W}\left(L \Gamma_{\mu \alpha}^{\sigma} \widehat{K}^{\alpha} n_{\sigma}+(K n)^{-1} \omega_{\mu}(K n)-\widehat{K} \omega_{\mu}\right) \Delta^{\mu \nu}\right\rangle= \\
= & \frac{1}{16 \pi^{2}(d-4)} \operatorname{tr}\left\langle\frac { 1 } { 2 } L ( L - 1 ) p _ { \nu } \widehat { W } \left((L-2) \Gamma_{\mu \alpha}^{\sigma} \widehat{K}^{\mu \nu \alpha} n_{\sigma}+\Gamma_{\mu \alpha}^{\mu} \widehat{K}^{\nu \alpha}+\right.\right. \\
& \left.+\Gamma_{\mu \alpha}^{\nu} \widehat{K}^{\mu \alpha}-\widehat{K}^{\mu \nu} \omega_{\mu}\right)+L p_{\nu} \widehat{W}\left((L-1) \Gamma_{\mu \alpha}^{\sigma} \widehat{K}^{\mu \alpha} n_{\sigma}+\right. \\
& \left.\left.+\Gamma_{\mu \alpha}^{\mu} \widehat{K}^{\alpha}-\widehat{K}^{\mu} \omega_{\mu}\right) \Delta^{\nu}+p_{\nu} \widehat{W}\left(L \Gamma_{\mu \alpha}^{\sigma} \widehat{K}^{\alpha} n_{\sigma}-\omega_{\mu}\right) \Delta^{\mu \nu}\right\rangle .
\end{aligned}
$$

(Возникновение данного выражения может быть интерпретировано так: результат должен быть обшекоординатно-инвариантен, поэтому в (24) необходимо заменить обычные производные на ковариантные и вычесть при этом лишние слагаемые из других диаграмм.)

Складывая (25) с (19) и (21), получаем окончательное выражение:

$$
\begin{aligned}
& \frac{1}{16 \pi^{2}(d-4)} \operatorname{tr}\left\langle\frac{1}{3} L \widehat{W} \widehat{K}^{\alpha} \Delta^{\mu \nu} n_{\sigma}\left(p_{\alpha} \Gamma_{\mu \nu}^{\sigma}-p_{\nu} \Gamma_{\mu \alpha}^{\sigma}\right)+\frac{1}{6} L(L-1) \times\right. \\
& \quad \times \widehat{W} \widehat{K}^{\mu \nu} \Delta^{\alpha} n_{\sigma}\left(p_{\alpha} \Gamma_{\mu \nu}^{\sigma}-p_{\nu} \Gamma_{\mu \alpha}^{\sigma}\right)+\frac{1}{6} L(L-1) \widehat{W} \widehat{K}^{\mu \nu}\left(p_{\sigma} \Gamma_{\mu \nu}^{\sigma}-p_{\mu} \Gamma_{\nu \sigma}^{\sigma}\right)- \\
& \left.\quad-\frac{1}{6} L \widehat{W} \widehat{K}^{\mu} \Delta^{\nu}\left(p_{\sigma} \Gamma_{\mu \nu}^{\sigma}-p_{\mu} \Gamma_{\nu \sigma}^{\sigma}\right)-\frac{1}{2} L^{2} \widehat{W}\left(p_{\mu} \omega_{\nu}-p_{\nu} \omega_{\mu}\right) \widehat{K}^{\mu} \widehat{K}^{\nu}\right\rangle
\end{aligned}
$$

которое может быть (в отличие от (19) и (21) !) представлено как первый неисчезающий член в разложении по слабому гравитационному полю от следующего ковариантного выражения:

$$
\begin{gathered}
\frac{1}{16 \pi^{2}(d-4)} \operatorname{tr}\left\langle-\frac{1}{2} L^{2} \widehat{W} F_{\mu \nu} \widehat{K}^{\mu} \widehat{K}^{\nu}+\frac{1}{6} L(L-1) \widehat{W} \widehat{K}^{\mu \nu} R_{\mu \nu}+\frac{1}{6} L^{2} \widehat{W}^{\mu} \widehat{K}^{\nu} R_{\mu \nu}+\right. \\
\left.+\frac{1}{3} L \widehat{W} \widehat{K}^{\alpha} \Delta^{\mu \nu} n_{\sigma} R_{\mu \alpha \nu}^{\sigma}+\frac{1}{6} L(L-1) \widehat{W} \widehat{K}^{\mu \nu} \Delta^{\alpha} n_{\sigma} R_{\mu \alpha \nu}^{\sigma}\right\rangle
\end{gathered}
$$

Оно и является ответом для рассматриваемых диаграмм. Далее мы постараемся записать данное выражение в наиболее компактной форме. Вначале учтем, что согласно правилам, сформулированным в приложении,

$$
\begin{aligned}
& -\frac{1}{2} L(L-1) \widehat{W} \widehat{K}^{\mu \nu} R_{\mu \nu}+L^{2} \widehat{W} \widehat{K}^{\mu} \widehat{K}^{\nu} R_{\mu \nu}= \\
& \quad \widehat{W} \Delta^{\mu \nu} R_{\mu \nu}=\frac{1}{2}(L-2)(L-3) \widehat{W}^{\mu \nu} R_{\mu \nu}
\end{aligned}
$$


и, следовательно, результат можно переписать в виде

$$
\begin{aligned}
& \frac{1}{16 \pi^{2}(d-4)} \operatorname{tr}\left\langle-\frac{1}{2} L^{2} \widehat{W} \widehat{K}^{\mu} F_{\mu \nu} \widehat{K}^{\nu}+\frac{1}{3} L \widehat{W} \widehat{K}^{\alpha} \Delta^{\mu \nu} n_{\sigma} R_{\mu \alpha \nu}^{\sigma}+\right. \\
& +\frac{1}{6} L(L-1) \widehat{W} \widehat{K}^{\mu \nu} \Delta^{\alpha} n_{\sigma} R_{\mu \alpha \nu}^{\sigma}+\frac{1}{12}(L-2)(L-3) \widehat{W}^{\mu \nu} R_{\mu \nu}+ \\
& \left.\quad+\frac{1}{4} L(L-1) \widehat{W} \widehat{K}^{\mu \nu} R_{\mu \nu}\right\rangle .
\end{aligned}
$$

Для дальнейшего упрошения записи результата оказывается удобным учесть следующие простые тождества:

$$
\begin{aligned}
\left(\nabla_{\mu} \nabla_{\nu}-\nabla_{\nu} \nabla_{\mu}\right)(K n)= & L n_{\rho} R_{\alpha \mu \nu}^{\rho}(K n)^{\alpha}+F_{\mu \nu}(K n)-(K n) F_{\mu \nu} ; \\
\left(\nabla_{\mu} \nabla_{\nu}-\nabla_{\nu} \nabla_{\mu}\right)(K n)^{\beta}= & (L-1) n_{\rho} R_{\alpha \mu \nu}^{\rho}(K n)^{\alpha \beta}+R^{\beta}{ }_{\alpha \mu \nu}(K n)^{\alpha}+ \\
& +F_{\mu \nu}(K n)^{\beta}-(K n)^{\beta} F_{\mu \nu} ; \\
\left(\nabla_{\mu} \nabla_{\nu}-\nabla_{\nu} \nabla_{\mu}\right)(K n)^{\beta \gamma=} & (L-2) n_{\rho} R^{\rho}{ }_{\alpha \mu \nu}(K n)^{\alpha \beta \gamma}+R^{\beta}{ }_{\alpha \mu \nu}(K n)^{\alpha \gamma}+ \\
& +R_{\alpha \mu \nu}^{\gamma}(K n)^{\alpha \beta}+F_{\mu \nu}(K n)^{\beta \gamma}-(K n)^{\beta \gamma} F_{\mu \nu}
\end{aligned}
$$

(в данном случае нам будет необходимо только первое из них; при анализе других слагаемых используются также и остальные).

Окончательно результат может быть представлен в виде

$$
\begin{aligned}
& \frac{1}{16 \pi^{2}(d-4)} \operatorname{tr}\left\langle-\frac{1}{2} L^{2} \widehat{W} \widehat{F}_{\mu \nu}(K n)^{\mu} \widehat{K}^{\nu}+\frac{1}{3} L \widehat{W} \widehat{K}^{\alpha} \Delta^{\mu \nu} n_{\sigma} R_{\mu \alpha \nu}^{\sigma}+\right. \\
& \left.+\frac{1}{3} L^{2}(L-1) \widehat{W} \widehat{K}^{\mu \nu} \widehat{K}^{\alpha} n_{\sigma} R_{\mu \alpha \nu}^{\sigma}-\frac{1}{6}(L-2)(L-3) \widehat{W}^{\mu \nu} R_{\mu \nu}\right\rangle .
\end{aligned}
$$

Оставшиеся диаграммы рассматриваются аналогично. Однако необходимо заметить, что вычисление диаграмм, соответствующих членам, квадратичным по тензору кривизны $(R R)$, является весьма затруднительным. Данная проблема детально рассмотрена в $[8]$.

Собирая результаты вычислений для различных групп диаграмм, в итоге получаем

$$
\Gamma_{\infty}^{(1)}=\frac{1}{16 \pi^{2}(d-4)} \operatorname{tr} \int d^{4} x \sqrt{-g}\langle\text { Flat }+W R+S R+S S R+F F+F R+R R\rangle
$$

(Flat означает результат в случае плоского пространства). В формуле (32):

$$
\begin{aligned}
\text { Flat }= & \frac{1}{4} \widehat{S}^{4}-\widehat{W} \widehat{S}^{2}+\frac{1}{2} \widehat{W}^{2}+\widehat{S} \widehat{N}-\widehat{M}+(L-2) \nabla_{\mu} \widehat{S} \widehat{W}^{\mu}-L \nabla_{\mu} \widehat{S} \widehat{W} \widehat{K}^{\mu}+ \\
& +\frac{1}{3}\left((L-1) \nabla_{\mu} \widehat{S}^{\mu} \widehat{S}^{2}-L \nabla_{\mu} \widehat{S} \widehat{K}^{\mu} \widehat{S}^{2}-(L-1) \nabla_{\mu} \widehat{S} \widehat{S}^{\mu}+L \nabla_{\mu} \widehat{S} \widehat{S}^{2} \widehat{K}^{\mu}\right)- \\
& -\frac{1}{2} \nabla_{\mu} \widehat{S} \nabla_{\nu} \widehat{S} \Delta^{\mu \nu}-\frac{1}{4}(L-1)(L-2) \nabla_{\mu} \widehat{S} \nabla_{\nu} \widehat{S}^{\mu \nu}+ \\
& +\frac{1}{4} L(L-1) \nabla_{\mu} \widehat{S} \nabla_{\nu} \widehat{S}^{\nu} \widehat{K}^{\mu}+\frac{1}{4} L(L-1) \nabla_{\nu} \widehat{S} \nabla_{\mu} \widehat{S}^{\nu} \widehat{K}^{\mu}
\end{aligned}
$$


$W R=-\frac{1}{2} L^{2} \widehat{W} \widehat{F}_{\mu \nu}(K n)^{\mu} \widehat{K}^{\nu}+\frac{1}{3} L \widehat{W} \widehat{K}^{\alpha} \Delta^{\mu \nu} n_{\sigma} R_{\mu \alpha \nu}^{\sigma}+\frac{1}{3} L^{2}(L-1) \widehat{W} \times$ $\times \widehat{K}^{\mu \nu} \widehat{K}^{\alpha} n_{\sigma} R_{\mu \alpha \nu}^{\sigma}-\frac{1}{6}(L-2)(L-3) \widehat{W}^{\mu \nu} R_{\mu \nu}$,

$$
\begin{aligned}
S R= & -\frac{1}{12}(L-1)(L-2)(L-3) \widehat{S}^{\alpha \mu \nu} \nabla_{\alpha} R_{\mu \nu}-\frac{1}{12} L^{2}(L-1)(L-2) \widehat{S} \widehat{K}^{\mu \nu \alpha} \widehat{K}^{\beta} n_{\sigma} \times \\
& \times \nabla_{\alpha} R_{\mu \beta \nu}^{\sigma}-\frac{1}{6} L^{2}(L-1) \widehat{S} \nabla_{\mu} \hat{F}_{\alpha \nu}(K n)^{\mu \nu} \widehat{K}^{\alpha}+\frac{2}{3} L \widehat{S} \nabla_{\mu} \hat{F}_{\nu \alpha}(K n)^{\alpha} \Delta^{\mu \nu}+ \\
& +L(L-1) \widehat{S} \widehat{K}^{\mu \nu} \Delta^{\alpha \beta} n_{\sigma}\left(\frac{5}{12} \nabla_{\alpha} R_{\nu \beta \mu}^{\sigma}-\frac{1}{12} \nabla_{\mu} R_{\alpha \nu \beta}^{\sigma}\right)- \\
& -\frac{1}{2} L \widehat{S} \widehat{K}^{\beta} \Delta^{\mu \nu \alpha} n_{\sigma} \nabla_{\alpha} R_{\mu \beta \nu}^{\sigma},
\end{aligned}
$$

$$
\begin{aligned}
S S R= & \frac{1}{6} \widehat{S} \widehat{S} \Delta^{\mu \nu} R_{\mu \nu}-\frac{1}{2} L(L-1) \widehat{S} \widehat{S}^{\mu} \widehat{F}_{\mu \nu} \widehat{K}^{\nu}+\frac{1}{2} L^{2} \widehat{S} \widehat{S} \widehat{F}_{\mu \nu}(K n)^{\mu} \widehat{K}^{\nu}+ \\
& +\frac{1}{12}(L-1)(L-2) \widehat{S} \widehat{S}^{\mu \nu} R_{\mu \nu}+\frac{1}{3} L(L-1) \widehat{S} \widehat{S}^{\mu} \widehat{K}^{\nu} R_{\mu \nu}-\frac{1}{6} L(L-1) \times \\
& \times(L-2) \widehat{S} \widehat{S}^{\mu \nu} \widehat{K}^{\alpha} n_{\sigma} R_{\mu \alpha \nu}^{\sigma}+\frac{1}{3}(L-1) \widehat{S} \widehat{S}^{\alpha} \Delta^{\mu \nu} n_{\sigma} R_{\mu \alpha \nu}^{\sigma}-\frac{1}{3} L^{2}(L-1) \times \\
& \times \widehat{S} \widehat{S} \widehat{K}^{\mu \nu} \widehat{K}^{\alpha} n_{\sigma} R_{\mu \alpha \nu}^{\sigma}-\frac{1}{3} L \widehat{S} \widehat{S} \widehat{K}^{\alpha} \Delta^{\mu \nu} n_{\sigma} R_{\mu \alpha \nu}^{\sigma} \\
F F= & -\frac{1}{24} L^{2}(L-1)^{2} \widehat{K}^{\mu \nu} F_{\mu \alpha} \widehat{K}^{\alpha \beta} F_{\nu \beta}+\frac{1}{24} L^{2} \widehat{K}^{\mu} F_{\beta \nu} \Delta^{\alpha \beta} \widehat{K}^{\nu} F_{\alpha \mu}- \\
& \quad-\frac{5}{24} L^{2} \widehat{K}^{\mu} F_{\beta \mu} \Delta^{\alpha \beta} \widehat{K}^{\nu} F_{\alpha \nu}-\frac{1}{48} L^{2}(L-1) \widehat{K}^{\mu} F_{\beta \nu} \Delta^{\nu} \widehat{K}^{\alpha \beta} F_{\alpha \mu}- \\
& \quad-\frac{1}{48} L^{2}(L-1) \widehat{K}^{\mu} F_{\beta \mu} \Delta^{\nu} \widehat{K}^{\alpha \beta} F_{\alpha \nu}
\end{aligned}
$$

$$
\begin{aligned}
F R= & \frac{1}{40} L^{2}(L-1)(L-2) \Delta^{\mu} \widehat{K}^{\nu} \widehat{K}^{\alpha \beta \gamma} F_{\mu \alpha} n_{\sigma} R_{\gamma \beta \nu}^{\sigma}-L^{2}(L-1)(L-2) \times \\
& \times \Delta^{\nu} \widehat{K}^{\alpha \beta \gamma} \widehat{K}^{\mu} n_{\sigma}\left(\frac{1}{60} R_{\beta \gamma \mu}^{\sigma} F_{\alpha \nu}+\frac{1}{12} R_{\beta \gamma \nu}^{\sigma} F_{\alpha \mu}\right)+L^{2}(L-1)^{2} \Delta^{\alpha} \times \\
& \times \widehat{K}^{\beta \gamma} \widehat{K}^{\mu \nu} n_{\sigma}\left(\frac{1}{60} R_{\beta \mu \gamma}^{\sigma} F_{\alpha \nu}+\frac{1}{20} R_{\alpha \mu \gamma}^{\sigma} F_{\nu \beta}+\frac{1}{15} R_{\gamma \mu \alpha}^{\sigma} F_{\nu \beta}+\right. \\
& \left.+\frac{1}{60} R_{\mu \nu \gamma}^{\sigma} F_{\alpha \beta}\right)+L^{2}(L-1) \Delta^{\alpha \beta} \widehat{K}^{\gamma \delta} \widehat{K}^{\mu} n_{\sigma}\left(\frac{4}{15} R_{\delta \beta \gamma}^{\sigma} F_{\alpha \mu}-\right. \\
& \left.-\frac{1}{30} R_{\beta \delta \alpha}^{\sigma} F_{\gamma \mu}-\frac{1}{15} R_{\alpha \gamma \mu}^{\sigma} F_{\beta \delta}-\frac{1}{30} R_{\gamma \alpha \mu}^{\sigma} F_{\beta \delta}\right)+ \\
& +L^{2}(L-1) \Delta^{\alpha \beta} \widehat{K}^{\gamma} \widehat{K}^{\mu \nu} n_{\sigma}\left(\frac{7}{60} R_{\alpha \beta \mu}^{\sigma} F_{\gamma \nu}-\frac{11}{60} R_{\beta \mu \gamma}^{\sigma} F_{\alpha \nu}+\right. \\
& \left.+\frac{1}{5} R_{\mu \alpha \gamma}^{\sigma} F_{\beta \nu}+\frac{1}{60} R_{\mu \alpha \nu}^{\sigma} F_{\gamma \beta}\right)+L^{2} \Delta^{\mu \alpha \beta} \widehat{K}^{\gamma} \widehat{K}^{\nu} n_{\sigma} \times \\
& \times\left(\frac{7}{20} R_{\alpha \gamma \beta}^{\sigma} F_{\nu \mu}+\frac{1}{10} R_{\alpha \beta \nu}^{\sigma} F_{\gamma \mu}\right),
\end{aligned}
$$




$$
\begin{aligned}
& R R=\frac{1}{10} L^{2} \widehat{K}^{\delta} \Delta^{\mu \nu \alpha \beta} \widehat{K}^{\gamma} n_{\sigma} n_{\rho} R^{\sigma}{ }_{\alpha \beta \gamma} R^{\rho}{ }_{\mu \nu \delta}+L^{2}(L-1)^{2}(L-2) \widehat{K}^{\beta \gamma \delta} \Delta^{\alpha} \widehat{K}^{\mu \nu} n_{\sigma} n_{\rho} \times \\
& \times\left(\frac{2}{45} R_{\alpha \delta \nu}^{\rho} R_{\beta \mu \gamma}^{\sigma}-\frac{1}{120} R_{\delta \alpha \nu}^{\rho} R_{\beta \mu \gamma}^{\sigma}\right)+L^{2}(L-1) \widehat{K}^{\delta} \Delta^{\alpha \beta \gamma} \widehat{K}^{\mu \nu} n_{\sigma} n_{\rho} \times \\
& \times\left(-\frac{1}{10} R_{\mu \gamma \nu}^{\rho} R_{\alpha \delta \beta}^{\sigma}+\frac{1}{15} R_{\delta \alpha \nu}^{\rho} R_{\beta \mu \gamma}^{\sigma}+\frac{1}{60} R_{\beta \delta \nu}^{\rho} R_{\gamma \mu \alpha}^{\sigma}\right)+L^{2}(L-1)^{2} \times \\
& \times \widehat{K}^{\gamma \delta} \Delta^{\alpha \beta} \widehat{K}^{\mu \nu} n_{\sigma} n_{\rho} \times\left(-\frac{1}{20} R^{\rho}{ }_{\mu \beta \nu} R_{\delta \alpha \gamma}^{\sigma}+\frac{1}{180} R^{\rho}{ }_{\alpha \nu \beta} R^{\sigma}{ }_{\gamma \delta \mu}-\right. \\
& -\frac{7}{360} R_{\mu \gamma \nu}^{\rho} R_{\alpha \delta \beta}^{\sigma}-\frac{1}{240} R_{\delta \beta \nu}^{\rho} R_{\gamma \alpha \mu}^{\sigma}-\frac{1}{120} R_{\beta \gamma \nu}^{\rho} R_{\alpha \delta \mu}^{\sigma-} \\
& \left.-\frac{1}{30} R_{\delta \beta \nu}^{\rho} R_{\alpha \gamma \mu}^{\sigma}\right)+L^{2}(L-1)(L-2) \widehat{K}^{\delta} \Delta^{\mu \nu} \widehat{K}^{\alpha \beta \gamma} n_{\sigma} n_{\rho} \times \\
& \times\left(-\frac{1}{30} R_{\gamma \nu \beta}^{\rho} R_{\alpha \delta \mu}^{\sigma}-\frac{1}{180} R_{\mu \gamma \nu}^{\rho} R_{\alpha \beta \delta}^{\sigma}+\frac{1}{180} R_{\mu \gamma \delta}^{\rho} R_{\alpha \beta \nu}^{\sigma}\right)+ \\
& +L^{2}(L-1)^{2}(L-2) \widehat{K}^{\mu \nu} \Delta^{\delta} \widehat{K}^{\alpha \beta \gamma} n_{\sigma} n_{\rho} \times \\
& \times\left(\frac{1}{45} R_{\mu \gamma \nu}^{\rho} R_{\alpha \beta \delta}-\frac{1}{80} R_{\beta \nu \gamma}^{\rho} R_{\mu \alpha \delta}^{\sigma}+\frac{1}{90} R_{\beta \nu \gamma}^{\rho} R_{\delta \alpha \mu}^{\sigma}\right)+ \\
& +L^{2}(L-1) \widehat{K}^{\mu \nu} \Delta^{\alpha \beta \gamma} \widehat{K}^{\delta} n_{\sigma} n_{\rho}\left(\frac{7}{120} R_{\beta \gamma \nu}^{\rho} R_{\mu \alpha \delta}^{\sigma}-\frac{3}{40} R_{\beta \gamma \delta}^{\rho} R_{\mu \alpha \nu}^{\sigma}+\right. \\
& \left.+\frac{1}{120} R_{\delta \gamma \nu}^{\rho} R_{\alpha \beta \mu}^{\sigma}\right)+L^{2}(L-1)(L-2) \widehat{K}^{\alpha \beta \gamma} \Delta^{\mu \nu} \widehat{K}^{\delta} n_{\sigma} n_{\rho} \times \\
& \times\left(-\frac{1}{24} R_{\mu \gamma \nu}^{\rho} R_{\alpha \beta \delta}^{\sigma}-\frac{1}{180} R_{\nu \gamma \delta}^{\rho} R_{\alpha \beta \mu}^{\sigma}-\frac{1}{360} R_{\delta \gamma \nu}^{\rho} R_{\alpha \beta \mu}^{\sigma}\right)- \\
& -\frac{1}{120} L^{2}(L-1)(L-2)(L-3) \widehat{K}^{\mu \nu \alpha \beta} \Delta^{\delta} \widehat{K}^{\gamma} n_{\sigma} n_{\rho} R_{\alpha \beta \gamma}^{\rho} R_{\mu \nu \delta}^{\sigma}- \\
& -\frac{1}{80} L^{2}(L-1)^{2}(L-2)(L-3) \widehat{K}^{\alpha \beta \gamma \delta} \widehat{K}^{\mu \nu} n_{\sigma} n_{\rho} R_{\beta \gamma \mu}^{\rho} R_{\alpha \delta \nu}^{\sigma}+ \\
& +L^{2} \widehat{K}^{\mu} \Delta^{\alpha \beta \gamma} \widehat{K}^{\nu} n_{\rho}\left(-\frac{1}{8} R_{\beta \gamma} R_{\nu \alpha \mu}^{\rho}+\frac{3}{20} R_{\beta \gamma} R^{\rho}{ }_{\mu \alpha \nu}+\frac{3}{40} R_{\alpha \mu} R_{\beta \gamma \nu}^{\rho}+\right. \\
& \left.+\frac{1}{40} R_{\beta \gamma \mu}^{\sigma} R_{\nu \alpha \sigma}^{\rho}-\frac{3}{20} R_{\alpha \beta \mu}^{\sigma} R_{\gamma \nu \sigma}^{\rho}+\frac{1}{10} R_{\alpha \beta \nu}^{\sigma} R_{\gamma \mu \sigma}^{\rho}\right)+ \\
& +L^{2}(L-1) \widehat{K}^{\gamma} \Delta^{\alpha \beta} \widehat{K}^{\mu \nu} n_{\rho}\left(\frac{1}{20} R_{\alpha \nu} R_{\gamma \beta \mu}^{\rho}+\frac{1}{20} R_{\alpha \gamma} R^{\rho}{ }_{\mu \beta \nu}+\frac{1}{10} R_{\alpha \beta} R_{\mu \gamma \nu}^{\rho}+\right. \\
& \left.+\frac{1}{20} R_{\alpha \nu \gamma}^{\sigma} R_{\sigma \beta \mu}^{\rho}-\frac{1}{60} R_{\mu \alpha \nu}^{\sigma} R_{\beta \sigma \gamma}^{\rho}+\frac{1}{10} R_{\alpha \beta \gamma}^{\sigma} R_{\mu \sigma \nu}^{\rho}-\frac{1}{12} R_{\alpha \beta \nu}^{\sigma} R_{\mu \sigma \gamma}^{\rho}\right)+ \\
& +L^{2}(L-1)^{2} \widehat{K}^{\alpha \beta} \Delta^{\gamma} \widehat{K}^{\mu \nu} n_{\rho}\left(\frac{1}{60} R_{\alpha \mu} R_{\beta \nu \gamma}^{\rho}-\frac{1}{20} R_{\alpha \mu} R_{\gamma \nu \beta}^{\rho}+\right. \\
& +\frac{1}{120} R_{\alpha \beta} R^{\rho}{ }_{\mu \nu \gamma}+\frac{3}{40} R_{\alpha \gamma} R_{\nu \beta \mu}^{\rho}+\frac{1}{20} R_{\gamma \mu \alpha}^{\sigma} R_{\nu \sigma \beta}^{\rho}+\frac{1}{120} R_{\alpha \mu \gamma}^{\sigma} R_{\beta \nu \sigma^{-}} \\
& \left.-\frac{1}{40} R_{\alpha \mu \gamma}^{\sigma} R_{\sigma \nu \beta}^{\rho}+\frac{1}{40} R_{\alpha \mu \beta}^{\sigma} R_{\sigma \nu \gamma}^{\rho}-\frac{1}{20} R_{\alpha \mu \beta}^{\sigma} R_{\gamma \nu \sigma}^{\rho}-\frac{1}{40} R_{\mu \beta \nu}^{\sigma} R_{\gamma \sigma \alpha}^{\rho}\right)+
\end{aligned}
$$




$$
\begin{aligned}
& +L^{2}(L-1) \widehat{K}^{\alpha \beta} \Delta^{\mu \nu} \widehat{K}^{\gamma} n_{\rho}\left(\frac{1}{20} R_{\mu \nu \beta}^{\sigma} R_{\gamma \sigma \alpha}^{\rho}-\frac{7}{60} R_{\beta \mu \alpha}^{\sigma} R_{\gamma \nu \sigma}^{\rho}+\right. \\
& +\frac{1}{20} R_{\beta \mu \alpha}^{\sigma} R_{\sigma \nu \gamma}^{\rho}+\frac{1}{10} R_{\mu \beta \gamma}^{\sigma} R_{\nu \alpha \sigma}^{\rho}+\frac{1}{60} R_{\beta \mu \gamma}^{\sigma} R_{\alpha \nu \sigma}^{\rho}+\frac{7}{120} R_{\alpha \beta} R_{\nu \gamma \mu}^{\rho}+ \\
& \left.+\frac{11}{60} R_{\beta \mu} R_{\nu \alpha \gamma}^{\rho}\right)+L^{2}(L-1)(L-2) \widehat{K}^{\alpha \beta \gamma} \Delta^{\mu} \widehat{K}^{\nu} n_{\rho}\left(\frac{7}{240} R_{\alpha \beta} R_{\gamma \mu \nu}^{\rho}+\right. \\
& +\frac{7}{240} R_{\alpha \nu} R_{\beta \gamma \mu}^{\rho}-\frac{1}{60} R_{\alpha \mu} R_{\beta \gamma \nu}^{\rho}-\frac{1}{24} R_{\alpha \beta \nu}^{\sigma} R_{\sigma \gamma \mu}^{\rho}+\frac{1}{15} R_{\alpha \beta \nu}^{\sigma} R_{\mu \gamma \sigma}^{\rho}+ \\
& \left.+\frac{1}{40} R_{\alpha \beta \mu}^{\sigma} R_{\sigma \gamma \nu}^{\rho}+\frac{1}{40} R_{\beta \gamma} R_{\nu \mu \alpha}^{\rho}+\frac{1}{48} R_{\beta \gamma \mu}^{\sigma} R_{\nu \alpha \sigma}^{\rho}\right)+ \\
& +L^{2}(L-1)^{2}(L-2) \widehat{K}^{\alpha \beta \gamma} \widehat{K}^{\mu \nu} n_{\rho}\left(-\frac{7}{240} R_{\alpha \mu} R_{\beta \gamma \nu}^{\rho}+\frac{1}{240} R_{\beta \gamma} R_{\mu \alpha \nu}^{\rho}{ }_{\mu \alpha}\right. \\
& \left.-\frac{1}{40} R_{\alpha \mu \beta}^{\sigma} R_{\nu \gamma \sigma}^{\rho}\right)+L(L-1)(L-2)(L-3) \widehat{K}^{\mu \nu \alpha \beta} \times \\
& \times\left(\frac{1}{180} R_{\mu \nu} R_{\alpha \beta}+\frac{7}{720} R_{\alpha \beta \rho}^{\sigma} R_{\mu \nu \sigma}^{\rho}\right),
\end{aligned}
$$

где выражения для величин $\Delta^{\mu}-\Delta^{\mu \nu \alpha \beta}$ приведены в (П. 10).

Несмотря на громоздкость полученных результатов их обработка может быть быстро и эффективно произведена с использованием вычислительных машин, например при помоши тензорного пакета [9] для системы REDUCE [10]. Результаты соответствуюших вычислений для ряда частных случаев приводятся в следующем разделе.

В качестве замечания необходимо также отметить, что при получении результата (32) отбрасывались полные производные, поэтому при вычислениях в конкретных случаях всегда необходимо вьполнять подстановки

$$
R_{\alpha \beta \mu \nu} R^{\alpha \beta \mu \nu}=2 R_{\alpha \beta \mu \nu} R^{\alpha \mu \beta \nu} \rightarrow 4 R_{\mu \nu} R^{\mu \nu}-R^{2}
$$

(здесь мы приняли во внимание, что выражение $R_{\mu \nu \alpha \beta} R^{\mu \nu \alpha \beta}-4 R_{\mu \nu} R^{\mu \nu}+R^{2}$ является полной производной).

\section{4. ЧАСТНЫЕ СЛУЧАИ}

4.1. Минимальный оператор. Минимальный оператор порядка $2 l$ (в форме записи с симметризованным кинетическим членом) может быть рассмотрен как частный случай неминимального, если $L=2 l$ и $K^{\mu \nu \ldots \alpha}{ }_{i}^{j}=K_{0}{ }^{\mu \nu \ldots \alpha} \delta_{i}{ }^{j}$, где $K_{0}$ был определен формулой (5). После подстановки данной величины в (27) с использованием (11) приходим к выражению

$$
\begin{aligned}
\Gamma_{\infty}^{(1)}= & \frac{1}{16 \pi^{2}(d-4)} \operatorname{tr} \int d^{4} x \sqrt{-g}\left\langle(l-1) \nabla_{\mu} \widehat{S} \widehat{W}^{\mu}-\frac{(2 l-1)}{2} \nabla_{\mu} \widehat{S}^{\mu} \widehat{W}+\right. \\
& +\frac{(2 l-1)}{3} \nabla_{\mu} \widehat{S}^{\mu} \widehat{S}^{2}-\frac{(2 l-1)}{3} \nabla_{\mu} \widehat{S} \widehat{S} \widehat{S}^{\mu}-\frac{(2 l-1)\left(l^{2}-1\right)}{2(2 l+1)} \nabla_{\mu} \widehat{S}^{\mu \nu} \nabla_{\nu} \widehat{S}+ \\
& +\frac{(2 l-1)^{2} l}{4(2 l+1)} \nabla_{\mu} \widehat{S}^{\mu} \nabla_{\nu} \widehat{S}^{\nu}+\frac{l^{2}}{2(2 l+1)} \nabla_{\mu} \widehat{S} \nabla^{\mu} \widehat{S}+\widehat{S} \widehat{N}+\frac{1}{2} \widehat{W}^{2}+
\end{aligned}
$$




$$
\begin{aligned}
& +\frac{1}{4} \widehat{S}^{4}-\widehat{W} \widehat{S}^{2}-\widehat{M}-\frac{(2 l-3)(l-1)}{3(l+1)} \widehat{W}^{\mu \nu} R_{\mu \nu}+\frac{l^{2}}{3(l+1)} \widehat{W} R+ \\
& +\frac{(2 l-1)(2 l-3)(l-1)}{6(l+1)} \nabla_{\mu} \widehat{S}^{\mu \alpha \beta} R_{\alpha \beta}-\frac{l^{2}(2 l-1)}{6(l+1)} \nabla_{\mu} \widehat{S}^{\mu} R- \\
& -\frac{(2 l-1)^{2}(l-4)}{24(2 l+1)} R_{\mu \nu} \widehat{S}^{\mu} \widehat{S}^{\nu}+\frac{(2 l-1)(l-1)(l+2)}{6(2 l+1)} R_{\mu \nu} \widehat{S}^{\mu \nu} \widehat{S}- \\
& -\frac{l^{2}}{2(2 l+1)} \widehat{S}^{2} R-\frac{(2 l-1)(l-1)^{2}(l+2)}{12(2 l+1)} R_{\mu \nu \alpha \beta} \widehat{S}^{\mu \alpha} \widehat{S}^{\nu \beta}+ \\
& +\frac{l^{2}\left(3 l^{2}+4 l+2\right)}{1080} R^{2}+\frac{l^{2}\left(-7 l^{2}+4 l+12\right)}{540} R_{\mu \nu} R^{\mu \nu}+\frac{l^{3}}{12} F^{\mu \nu} F_{\mu \nu}+ \\
& \left.+\frac{(l+1)(2 l-1)^{2}}{4(2 l+1)} \widehat{S}^{\mu} \widehat{S}^{\nu} F_{\mu \nu}+\frac{l^{2}(2 l-1)}{3(l+1)} F^{\mu \nu} \nabla_{\mu} \widehat{S}_{\nu}\right\rangle
\end{aligned}
$$

которое совпадает с полученным в [6] для однопетлевого эффективного действия произвольного минимального оператора с симметризованным кинетическим членом

$$
\begin{aligned}
& D_{i}{ }^{j}=\delta_{i}{ }^{j} K_{0}{ }^{\mu_{1} \mu_{2} \ldots \mu_{2 l}} \nabla_{\mu_{1}} \nabla_{\mu_{2}} \ldots \nabla_{\mu_{2 l}}+S^{\mu_{1} \mu_{2} \ldots \mu_{2 l-1}}{ }_{i}^{j} \nabla_{\mu_{1}} \nabla_{\mu_{2}} \ldots \nabla_{\mu_{2 l-1}}+
\end{aligned}
$$

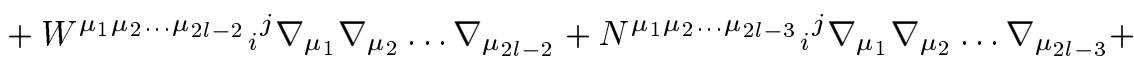

$$
\begin{aligned}
& +M^{\mu_{1} \mu_{2} \ldots \mu_{2 l-4}{ }_{i}{ }^{j}} \nabla_{\mu_{1}} \nabla_{\mu_{2}} \ldots \nabla_{\mu_{2 l-4}}+\cdots \text {. }
\end{aligned}
$$

Таким образом, установлено соответствие между результатами для минимального и неминимального операторов.

4.2. Векторное поле. В качестве другого примера рассмотрим оператор

$$
D=\square \delta_{\alpha}^{\beta}-\lambda \nabla_{\alpha} \nabla^{\beta}+P_{\alpha}^{\beta},
$$

причем $P_{\alpha \beta}=P_{\beta \alpha}$.

Запишем его в форме (4). Для этого необходимо произвести симметризашию второго слагаемого по $\alpha$ и $\beta$. Учитывая, что в данном случае квантовое поле является векторным, находим

$$
D=\left(g^{\mu \nu} \delta_{\alpha}{ }^{\beta}-\frac{\lambda}{2}\left(g^{\mu \beta} \delta_{\alpha}{ }^{\nu}+g^{\nu \beta} \delta_{\alpha}{ }^{\mu}\right)\right) \nabla_{\mu} \nabla_{\nu}+P_{\alpha}{ }^{\beta}+\frac{\lambda}{2} R_{\alpha}{ }^{\beta} .
$$

Таким образом, отличными от нуля являются только коэффициенты

$$
\begin{aligned}
K_{\alpha}^{\mu \nu}{ }^{\beta} & =g^{\mu \nu} \delta_{\alpha}{ }^{\beta}-\frac{\lambda}{2}\left(g^{\mu \beta} \delta_{\alpha}{ }^{\nu}+g^{\nu \beta} \delta_{\alpha}{ }^{\mu}\right), \\
W_{\alpha}{ }^{\beta} & =P_{\alpha}{ }^{\beta}+\frac{\lambda}{2} R_{\alpha}{ }^{\beta},
\end{aligned}
$$

и, следовательно,

$$
\begin{aligned}
(K n)_{\alpha}{ }^{\beta} & =\delta_{\alpha}{ }^{\beta}-\lambda n_{\alpha} n^{\beta}, \\
(K n)^{-1}{ }_{\alpha}{ }^{\beta} & =\delta_{\alpha}{ }^{\beta}+\gamma n_{\alpha} n^{\beta}, \quad \gamma \equiv \frac{\lambda}{1-\lambda} .
\end{aligned}
$$


Подставив данные величины в (32), приходим к следующему результату:

$$
\begin{aligned}
\Gamma_{\infty}^{(1)}= & \frac{1}{16 \pi^{2}(d-4)} \int d^{4} x \sqrt{-g}\left(\left(\frac{1}{24} \gamma^{2}+\frac{1}{4} \gamma+\frac{1}{2}\right) P_{\mu \nu} P^{\mu \nu}+\frac{1}{48} \gamma^{2} P^{2}+\right. \\
& +\left(\frac{1}{12} \gamma^{2}+\frac{1}{3} \gamma\right) R_{\mu \nu} P^{\mu \nu}+\left(\frac{1}{24} \gamma^{2}+\frac{1}{12} \gamma+\frac{1}{6}\right) R P+ \\
& \left.+\left(\frac{1}{24} \gamma^{2}+\frac{1}{12} \gamma-\frac{4}{15}\right) R_{\mu \nu} R^{\mu \nu}+\left(\frac{1}{48} \gamma^{2}+\frac{1}{12} \gamma+\frac{7}{60}\right) R^{2}\right)
\end{aligned}
$$

полностью совпадаюшему с выражениями, найденньми в работах [4] и [11] (здесь $P \equiv$ $\left.P_{\alpha}^{\alpha}\right)$.

Для проверки результата (32) было произведено также вычисление однопетлевых расходимостей для квадрата оператора (43), который записывается в виде

$$
\begin{aligned}
D_{\alpha}^{2}{ }^{\beta}= & \delta_{\alpha}{ }^{\beta} \square^{2}-\lambda \nabla_{\alpha} \nabla^{\beta} \square+2 P_{\alpha}^{\beta} \square-\lambda \square \nabla_{\alpha} \nabla^{\beta}+\lambda^{2} \nabla_{\alpha} \square \nabla^{\beta}-\lambda P_{\alpha}{ }^{\mu} \nabla_{\mu} \nabla^{\beta}- \\
& -\lambda P_{\mu}{ }^{\beta} \nabla_{\alpha} \nabla^{\mu}+\left(\square P_{\alpha}^{\beta}\right)+2\left(\nabla_{\mu} P_{\alpha}{ }^{\beta}\right) \nabla^{\mu}-\lambda\left(\nabla_{\alpha} \nabla^{\mu} P_{\mu}{ }^{\beta}\right)- \\
& -\lambda\left(\nabla_{\alpha} P_{\mu}{ }^{\beta}\right) \nabla^{\mu}-\lambda\left(\nabla_{\mu} P_{\mu}{ }^{\beta}\right) \nabla^{\alpha}+P_{\alpha}{ }^{\mu} P_{\mu}{ }^{\beta}
\end{aligned}
$$

и является неминимальным оператором четвертого порядка.

При помощи коммутации ковариантных производных он может быть представлен в виде (4), причем

$$
\begin{aligned}
& K^{\mu \nu \gamma \delta}{ }_{\alpha}^{\beta}=\delta_{\alpha}{ }^{\beta} \frac{1}{3}\left(g^{\mu \nu} g^{\gamma \delta}+g^{\mu \gamma} g^{\nu \delta}+g^{\mu \delta} g^{\nu \gamma}\right)+ \\
& +\frac{1}{12}\left(-2 \lambda+\lambda^{2}\right)\left(g^{\mu \nu} \delta_{\alpha}^{\gamma} g^{\beta \delta}+g^{\mu \nu} \delta_{\alpha}{ }^{\delta} g^{\beta \gamma}+g^{\mu \gamma} \delta_{\alpha}^{\nu} g^{\beta \delta}+g^{\mu \gamma} \delta_{\alpha}{ }^{\delta} g^{\beta \nu}+\right. \\
& +g^{\mu \delta} \delta_{\alpha}^{\nu} g^{\beta \gamma}+g^{\mu \delta} \delta_{\alpha}^{\gamma} g^{\beta \nu}+g^{\nu \gamma} \delta_{\alpha}^{\mu} g^{\beta \delta}+g^{\nu \gamma} \delta_{\alpha}{ }^{\beta} g^{\beta \mu}+ \\
& \left.+g^{\nu \delta} \delta_{\alpha}^{\mu} g^{\beta \gamma}+g^{\nu \delta} \delta_{\alpha}^{\gamma} g^{\beta \mu}+g^{\gamma \delta} \delta_{\alpha}^{\mu} g^{\beta \nu}+g^{\gamma \delta} \delta_{\alpha}{ }^{\nu} g^{\beta \mu}\right), \\
& S^{\mu \nu \gamma}{ }_{\alpha}^{\beta}=0 \\
& W_{\alpha}^{\mu \nu}{ }^{\beta}=2 P_{\alpha}{ }^{\beta} g^{\mu \nu}-\frac{\lambda}{2} P_{\alpha}^{\mu} g^{\nu \beta}-\frac{\lambda}{2} P_{\alpha}{ }^{\nu} g^{\mu \beta}-\frac{\lambda}{2} P^{\beta \mu} \delta_{\alpha}{ }^{\nu}-\frac{\lambda}{2} P^{\beta \nu} \delta_{\alpha}{ }^{\mu}+ \\
& +\frac{1}{6}\left(\lambda-2 \lambda^{2}\right)\left(R_{\alpha}^{\mu} g^{\nu \beta}+R_{\alpha}^{\nu} g^{\mu \beta}+R^{\beta \mu} \delta_{\alpha}{ }^{\nu}+R^{\beta \nu} \delta_{\alpha}{ }^{\mu}\right)+ \\
& +\frac{1}{6}\left(2 \lambda-\lambda^{2}\right)\left(R_{\alpha}^{\mu \beta \nu}+R_{\alpha}^{\nu \beta \mu}\right)+\frac{1}{2}\left(2 \lambda-\lambda^{2}\right) g^{\mu \nu} R_{\alpha}{ }^{\beta}-\frac{2}{3} R^{\mu \nu} \delta_{\alpha}{ }^{\beta}, \\
& M_{\alpha}^{\beta}=P_{\alpha \mu} P^{\mu \beta}+\frac{\lambda}{2} P_{\alpha \mu} R^{\mu \beta}+\frac{\lambda}{2} P_{\mu \nu} R_{\alpha}^{\mu}{ }^{\nu \beta}-\frac{1}{2} R_{\mu \nu \gamma \alpha} R^{\mu \nu \gamma \beta}+ \\
& +\frac{1}{6}\left(\lambda-2 \lambda^{2}\right) R_{\alpha \mu} R^{\mu \beta}+\frac{1}{12}\left(4 \lambda+7 \lambda^{2}\right) R_{\mu \alpha \nu}{ }^{\beta} R^{\mu \nu}+ \\
& +\frac{1}{4}\left(2 \lambda-\lambda^{2}\right) R_{\alpha \mu \nu \gamma} R^{\gamma \mu \nu \beta}
\end{aligned}
$$


(в данном случае $\left.(K n)^{-1} \alpha^{\beta}=\delta_{\alpha}{ }^{\beta}+\left(2 \gamma+\gamma^{2}\right) n_{\alpha} n^{\beta}\right)$.

Вычисления по формуле (32) привели к удвоенному результату (47), что и должно быть в силу очевидного соотношения $\operatorname{tr} \ln D^{2}=2 \operatorname{tr} \ln D$.

4.3. Гравитация в $\lambda$-калибровке. Рассмотрим эйнштейновскую теорию гравитации, описьваемую действием

$$
S=\int d^{4} x \sqrt{-g} R
$$

Оно инвариантно относительно общекоординатных преобразований, вследствие чего после разложения поля метрики на квантовую и классическую части: $g_{\mu \nu} \rightarrow g_{\mu \nu}+h_{\mu \nu}$, и разложения действия до второго порядка для поля $h_{\mu \nu}$ получаем оператор, имеюший нулевую моду,

$$
h_{\mu \nu} \rightarrow h_{\mu \nu}+\xi^{\alpha} \nabla_{\alpha} h_{\mu \nu}+\left(g_{\nu \alpha}+h_{\nu \alpha}\right) \nabla_{\mu} \xi^{\alpha}+\left(g_{\mu \alpha}+h_{\mu \alpha}\right) \nabla_{\nu} \xi^{\alpha} .
$$

Она может быть устранена при добавления слагаемых, фиксируюших обшекоординатные преобразования:

$$
S_{\mathrm{gf}}=-\frac{1}{2} \int d^{4} x \sqrt{-g} g_{\mu \nu} \chi^{\mu} \chi^{\nu}
$$

где

$$
\chi^{\mu}=\frac{1}{\sqrt{1+\lambda}}\left(g^{\mu \alpha} \nabla^{\beta} h_{\alpha \beta}-\frac{1}{2} g^{\alpha \beta} \nabla^{\mu} h_{\alpha \beta}\right) .
$$

После этого для второй вариации действия имеем

$$
\begin{aligned}
\frac{\delta^{2} S}{\delta h_{\alpha \beta} \delta h_{\mu \nu}}= & \sqrt{-g} C^{\alpha \beta, \gamma \delta}\left(\delta_{\gamma \delta}^{\mu \nu} \square-\frac{\lambda}{2(1+\lambda)}\left(\delta_{\gamma}^{\mu} \nabla_{\delta} \nabla^{\nu}+\right.\right. \\
& \left.+\delta_{\gamma}^{\nu} \nabla_{\delta} \nabla^{\mu}+\delta_{\delta}^{\mu} \nabla_{\gamma} \nabla^{\nu}+\delta_{\delta}^{\nu} \nabla_{\gamma} \nabla^{\mu}\right)+ \\
& \left.+\frac{\lambda}{2(1+\lambda)} g^{\mu \nu}\left(\nabla_{\gamma} \nabla_{\delta}+\nabla_{\delta} \nabla_{\gamma}\right)+P_{\gamma \delta}{ }^{\mu \nu}\right),
\end{aligned}
$$

где

$$
\begin{aligned}
\delta_{\alpha \beta}^{\mu \nu}= & \frac{1}{2}\left(\delta_{\alpha}^{\mu} \delta_{\beta}^{\nu}+\delta_{\alpha}^{\nu} \delta_{\beta}^{\mu}\right), \quad C^{\alpha \beta, \gamma \delta} \equiv \frac{1}{4}\left(g^{\alpha \gamma} g^{\beta \delta}+g^{\alpha \delta} g^{\beta \gamma}-g^{\alpha \beta} g^{\gamma \delta}\right) \\
P_{\gamma \delta}{ }^{\mu \nu}= & R_{\gamma}{ }^{\mu} \delta^{\nu}+R_{\gamma}{ }^{\nu} \delta^{\mu}+\frac{1}{2}\left(\delta_{\gamma}^{\mu} R_{\delta}^{\nu}+\delta_{\gamma}^{\nu} R_{\delta}^{\mu}+\delta_{\delta}^{\mu} R_{\gamma}{ }^{\nu}+\delta_{\delta}^{\nu} R_{\gamma}{ }^{\mu}\right)-g^{\mu \nu} R_{\gamma \delta}- \\
& -g_{\gamma \delta} R^{\mu \nu}-\delta_{\gamma \delta}^{\mu \nu} R+\frac{1}{2} g_{\gamma \delta} g^{\mu \nu} R
\end{aligned}
$$

Очевидно, что $\operatorname{tr} \ln \left(\sqrt{-g} C^{\alpha \beta, \mu \nu}\right)$ пропорционален $\delta(0)$ и в размерной регуляризации дает нулевой вклад. Поэтому в данном случае

$$
\begin{aligned}
D_{\alpha \beta}{ }^{\mu \nu}= & \delta_{\gamma \delta}^{\mu \nu} \square-\frac{\lambda}{2(1+\lambda)}\left(\delta_{\gamma}^{\mu} \nabla_{\delta} \nabla^{\nu}+\delta_{\gamma}^{\nu} \nabla_{\delta} \nabla^{\mu}+\delta_{\delta}^{\mu} \nabla_{\gamma} \nabla^{\nu}+\delta_{\delta}^{\nu} \nabla_{\gamma} \nabla^{\mu}\right)+ \\
& +\frac{\lambda}{2(1+\lambda)} g^{\mu \nu}\left(\nabla_{\gamma} \nabla_{\delta}+\nabla_{\delta} \nabla_{\gamma}\right)+P_{\gamma \delta}{ }^{\mu \nu}
\end{aligned}
$$


и, следовательно,

$$
\begin{gathered}
K_{\alpha \beta}^{\mu \nu}{ }^{\gamma \delta}=g^{\mu \nu} \delta_{\alpha \beta}{ }^{\gamma \delta}-\frac{\lambda}{4(1+\lambda)}\left(\delta_{\alpha}^{\gamma} \delta_{\beta}{ }^{\mu} g^{\delta \nu}+\delta_{\alpha}{ }^{\gamma} \delta_{\beta}{ }^{\nu} g^{\delta \mu}+\delta_{\alpha}{ }^{\delta} \delta_{\beta}{ }^{\mu} g^{\gamma \nu}+\right. \\
\left.+\delta_{\alpha}{ }^{\delta} \delta_{\beta}{ }^{\nu} g^{\gamma \mu}+\delta_{\beta}{ }^{\gamma} \delta_{\alpha}{ }^{\mu} g^{\delta \nu}+\delta_{\beta}{ }^{\gamma} \delta_{\alpha}{ }^{\nu} g^{\delta \mu}+\delta_{\beta}{ }^{\delta} \delta_{\alpha}{ }^{\mu} g^{\gamma \nu}+\delta_{\beta}{ }^{\delta} \delta_{\alpha}{ }^{\nu} g^{\gamma \mu}\right)+ \\
+\frac{\lambda}{2(1+\lambda)} g^{\gamma \delta}\left(\delta_{\alpha}{ }^{\mu} \delta_{\beta}{ }^{\nu}+\delta_{\alpha}{ }^{\nu} \delta_{\beta}{ }^{\mu}\right) \\
W_{\alpha \beta}{ }^{\gamma \delta}=P_{\alpha \beta}{ }^{\gamma \delta}-\frac{\lambda}{2(1+\lambda)}\left(R_{\alpha}{ }^{\gamma}{ }^{\delta}+R_{\alpha}{ }^{\delta}{ }^{\gamma}\right)+ \\
+\frac{\lambda}{4(1+\lambda)}\left(\delta_{\alpha}{ }^{\gamma} R_{\beta}{ }^{\delta}+\delta_{\alpha}{ }^{\delta} R_{\beta}{ }^{\gamma}+\delta_{\beta}{ }^{\gamma} R_{\alpha}{ }^{\delta}+\delta_{\beta}{ }^{\delta} R_{\alpha}{ }^{\gamma}\right) \\
(K n)^{-1}{ }_{\alpha \beta}^{\gamma \delta}=\delta_{\alpha \beta}^{\gamma \delta}+\frac{\lambda}{2}\left(\delta_{\alpha}{ }^{\gamma} n_{\beta} n^{\delta}+\delta_{\alpha}{ }^{\delta} n_{\beta} n^{\gamma}+\right. \\
\left.+\delta_{\beta}{ }^{\gamma} n_{\alpha} n^{\delta}+\delta_{\beta}{ }^{\delta} n_{\alpha} n^{\gamma}\right)-\lambda g^{\gamma \delta} n_{\alpha} n_{\beta}
\end{gathered}
$$

Вычисление однопетлевого эффективного действия производится согласно алгоритму (32). Для произвольного $P_{\mu \nu}{ }^{\alpha \beta}$, симметричного по каждой паре своих аргументов, был получен следующий результат:

$$
\begin{aligned}
& \frac{1}{16 \pi^{2}(d-4)} \int d^{4} x \sqrt{-g} \frac{1}{48}\left(\lambda^{2}\left(P^{\mu}{ }_{\mu \nu}{ }^{\nu}\right)^{2}+\left(-4 \lambda^{2}-12 \lambda\right) P_{\mu \nu \alpha}{ }^{\alpha} P_{\beta}{ }^{\beta \mu \nu}+\right. \\
& \quad+\left(4 \lambda^{2}+24 \lambda+24\right) P_{\alpha \beta \mu \nu} P^{\mu \nu \alpha \beta}+4 \lambda^{2} P_{\mu \nu \alpha \beta} P^{\nu \beta \mu \alpha}+4 \lambda^{2} P_{\mu \alpha \nu}{ }^{\alpha} P_{\beta}{ }^{\nu \beta \mu}+ \\
& \quad+2 \lambda^{2} P^{\alpha}{ }_{\alpha \mu \nu} P_{\beta}{ }^{\beta \mu \nu}-8 \lambda^{2} P_{\mu \alpha \nu}{ }^{\alpha} P_{\beta}{ }^{\beta \mu \nu}+\left(4 \lambda^{2}+8 \lambda+8\right) P_{\mu \nu}{ }^{\mu \nu} R- \\
& \quad-\left(4 \lambda^{2}+4 \lambda\right) P^{\mu}{ }_{\mu \nu}{ }^{\nu} R+4 \lambda^{2} P^{\mu \nu \alpha}{ }_{\alpha} R_{\mu \nu}+\left(8 \lambda^{2}+32 \lambda\right) P_{\alpha}{ }^{\mu \alpha \nu} R_{\mu \nu}+ \\
& \quad+\left(-4 \lambda^{2}+8 \lambda\right) P_{\alpha}{ }^{\alpha \mu \nu} R_{\mu \nu}+\left(-8 \lambda^{2}-48 \lambda\right) P_{\mu \nu \alpha \beta} R^{\mu \alpha \nu \beta}+ \\
& \left.\quad+\left(44 \lambda^{2}+32 \lambda-88\right) R_{\mu \nu} R^{\mu \nu}+\left(-4 \lambda^{2}+24 \lambda+28\right) R^{2}\right) .
\end{aligned}
$$

При подстановке в данное выражение $P_{\mu \nu}^{\alpha \beta}$ из (57) получаем, что основной вклад имеет вид

$$
\frac{1}{16 \pi^{2}(d-4)} \int d^{4} x \sqrt{-g}\left(\frac{1}{6}\left(4 \lambda^{2}+4 \lambda+7\right) R^{\mu \nu} R_{\mu \nu}+\frac{1}{12}\left(4 \lambda^{2}+7\right) R^{2}\right) .
$$

Кроме того, необходимо еше учесть вклад духов Фаддеева-Попова, лагранжиан которых получается с помощью бесконечно малого калибровочного преобразования $\chi^{\mu}$ и имеет вид

$$
L_{g h}=\bar{c}^{\alpha}\left(\delta^{\alpha}{ }_{\beta} \nabla^{\mu} \nabla_{\mu}+R_{\beta}^{\alpha}\right) c^{\beta} .
$$

Вклад духовых полей легко вычисляется по формуле (41) и имеет вид

$$
-2 \cdot \frac{1}{16 \pi^{2}(d-4)} \int d^{4} x \sqrt{-g}\left(\frac{7}{30} R_{\mu \nu} R^{\mu \nu}+\frac{17}{60} R^{2}\right)
$$


Окончательно, складывая (63) и (65), получаем результат

$$
\begin{aligned}
\Gamma_{\infty}^{(1)}= & \frac{1}{16 \pi^{2}(d-4)} \int d^{4} x \sqrt{-g}\left(\frac{1}{30}\left(20 \lambda^{2}+20 \lambda+21\right) R^{\mu \nu} R_{\mu \nu}+\right. \\
& \left.+\frac{1}{60}\left(20 \lambda^{2}+1\right) R^{2}\right)
\end{aligned}
$$

Формула (66) полностью совпадает с результатами, найденными в работах [12] и [4]. На уравнениях движения $R_{\mu \nu}=0$ данное выражение дает нулевой вклад (а следовательно, не зависит от выбора калибровки).

\section{5. ЗАКЛЮЧЕНИЕ}

В настоящей работе мы вычислили в обшем виде расходящуюся часть однопетлевого эффективного действия в искривленном пространстве-времени для произвольного неминимального оператора без каких-либо ограничений на его форму и порядок. Суть проделанной работы заключается в том, что для наиболее общего случая были выполнены основные операции, встречающиеся при вычислении фейнмановских диаграмм в искривленном пространстве, а именно: выполнение интегрирования по петлевому импульсу, суммирование всех расходящихся диаграмм с учетом их числовых коэффициентов и, наконец, переход от приближения слабого поля к ковариантному результату. После этого для вычисления расходящейся части остается подставить только конкретное выражение для второй вариации действия и выполнить ряд алгебраических операций.

K сожалению, полученный алгоритм является чрезвычайно громоздким, что весьма затрудняет его использование. Однако он может быть очень легко автоматизирован с использованием компьютеров, что создает ему определенные преимушества перед другими известными в литературе методами. Поэтому предложенный подход может быть успешно использован для проведения вычислений в неминимальных калибровках [12], в теориях, регуляризованных высшими производными [13], и в моделях с полями высших спинов.

В частности, при помощи тензорного пакета для системы аналитических вычислений REDUCE [9] мы вычислили контрчлены для ряда известных случаев. При этом наши результаты всегда оказывались в соответствии с ранее известными.

Приведем здесь время счета и затраты памяти для рассмотренных примеров (вычисления выполнены с использованием компютера IBM-486/DX-2/66/8Mb).

1. Вычисление $R R$ для частного случая минимального оператора произвольного порядка $l$ на основе общей формулы (32) для произвольного неминимального оператора: время вычислений - 17 с, затрачивается приблизительно 500 kb памяти.

2. Вычисление однопетлевых контрчленов для неминимального оператора с векторным квантовым полем (43): время - 220 с, используется около 3 Мb памяти. Для квадрата данного оператора требуется также $3 \mathrm{Mb}$ памяти, время вычислений составило $630 \mathrm{c.}$

3. Для гравитации в $\lambda$-калибровке требуется уже $8 \mathrm{Mb}$ оперативной памяти, а время вычислений увеличивается до 170 минут. 


\section{ПРИЛОЖЕНИЕ}

Рассмотрим интеграл $\int d^{d} k f(k)$ и предположим, что все слагаемые в нем имеют более чем логарифмическую степень расходимости и, следовательно, равны нулю (напомним, что используется размерная регуляризация).

Если произвести замену переменной $k_{\mu} \rightarrow k_{\mu}+p_{\mu}$, то расходящаяся часть при этом измениться не может. Вычислим ее явно, используя разложение в ряд по петлевому импульсу и удерживая логарифмически расходящиеся слагаемые.

Пусть $f(k)$ преобразуется при растяжении $k \rightarrow \alpha k$ как $f(k) \rightarrow \alpha^{-3} f(k)$. Тогда

$$
0=\left(\int d^{d} k f(k+p)\right)_{\infty}=\int d^{d} k \frac{1}{k^{4}} \delta_{\mu} f(n) p^{\mu}=\int d^{d} k \frac{1}{k^{4}}\left\langle\delta_{\mu} f(n)\right\rangle p^{\mu},
$$

где $\delta_{\mu} f(n)$ обозначает линейное по $p^{\mu}$ слагаемое в разложении $f(k+p)$ при $k_{\alpha}=n_{\alpha}$ :

$$
f(k+p) \equiv f(k)+\delta_{\mu} f(k) p^{\mu}+\delta_{\mu \nu} f(k) p^{\mu} p^{\nu}+\cdots
$$

Здесь мы предполагаем, что $\delta_{\mu \nu} f(n)$ является полностью симметричным по своим индексам. В силу произвольности $p^{\mu}$ заключаем, что

$$
\left\langle\delta_{\mu} f(n)\right\rangle=0
$$

Если $f(k) g(k) \rightarrow \alpha^{-3} f(k) g(k)$, то

$$
\left(\int d^{d} k g(k) f(k+p)\right)_{\infty}=\left(\int d^{d} k g(k-p) f(k)\right)_{\infty},
$$

и мы находим, что

$$
\left\langle g(n) \delta_{\mu} f(n)\right\rangle=-\left\langle\delta_{\mu} g(n) f(n)\right\rangle .
$$

В случае $f(k) g(k) \rightarrow \alpha^{-2} f(k) g(k)$ имеем

$$
\begin{aligned}
\left(\int d^{d} k g(k) f(k+p)\right)_{\infty} & =\left(\int d^{d} k g(k-p) f(k)\right)_{\infty}= \\
& =\int d^{d} k \frac{1}{k^{4}} g(n) \delta_{\mu \nu} f(n) p^{\mu} p^{\nu}= \\
& =\int d^{d} k \frac{1}{k^{4}} \delta_{\mu \nu} g(n) f(n) p^{\mu} p^{\nu}
\end{aligned}
$$

следовательно,

$$
\left\langle g(n) \delta_{\mu \nu} f(n)\right\rangle=\left\langle\delta_{\mu \nu} g(n) f(n)\right\rangle .
$$

Другие случаи рассматриваются аналогично.

При применении данных правил необходимо знать, чему равны коэффициенты разложения пропагатора неминимального оператора, выражения для которых многократно использовались при вычислении практически всех нетривиальных диаграмм. Для них 
были введены специальные обозначения, позволяющие значительно упростить результаты и облегчить вычисления:

$$
\begin{aligned}
(K(k+p))^{-1}= & (K k)^{-1}+\delta^{\mu}(K k)^{-1} p_{\mu}+\delta^{\mu \nu}(K k)^{-1} p_{\mu} p_{\nu}+ \\
& +\delta^{\mu \nu \alpha}(K k)^{-1} p_{\mu} p_{\nu} p_{\alpha}+\delta^{\mu \nu \alpha \beta}(K k)^{-1} p_{\mu} p_{\nu} p_{\alpha} p_{\beta}+\cdots \equiv \\
\equiv & \left(1+\frac{1}{k} \Delta^{\mu} p_{\mu}+\frac{1}{k^{2}} \Delta^{\mu \nu} p_{\mu} p_{\nu}+\frac{1}{k^{3}} \Delta^{\mu \nu \alpha} p_{\mu} p_{\nu} p_{\alpha}+\right. \\
& \left.+\frac{1}{k^{4}} \Delta^{\mu \nu \alpha \beta} p_{\mu} p_{\nu} p_{\alpha} p_{\beta}+\cdots\right)(K k)^{-1}
\end{aligned}
$$

где для удобства записи результатов мы вынесли $(K k)^{-1}$ за скобки (коэффициенты при высших степенях $p_{\mu}$ при расчетах расходяшейся части эффективного действия не требуются).

Для нахождения коэффициентов $\Delta^{\mu}-\Delta^{\mu \nu \alpha \beta}$ будем использовать следующее тождество:

$$
1_{i}^{j}=(K(k+p))_{i}{ }^{m}(K(k+p))^{-1}{ }^{j} .
$$

Разлагая его в ряд по $p_{\mu}$ и приравнивая коэффициенты при одинаковых степенях, получаем

$$
\begin{aligned}
\Delta^{\mu} \equiv & -L \widehat{K}^{\mu}, \\
\Delta^{\mu \nu} \equiv & -\frac{1}{2} L(L-1) \widehat{K}^{\mu \nu}+L^{2} \widehat{K}^{(\mu} \widehat{K}^{\nu)}, \\
\Delta^{\mu \nu \alpha} \equiv- & \frac{1}{6} L(L-1)(L-2) \widehat{K}^{\mu \nu \alpha}+\frac{1}{2} L^{2}(L-1) \widehat{K}^{(\mu \nu} \widehat{K}^{\alpha)}+\frac{1}{2} L^{2}(L-1) \times \\
& \times \widehat{K}^{(\alpha} \widehat{K}^{\mu \nu)}-L^{3} \widehat{K}^{(\mu} \widehat{K}^{\nu} \widehat{K}^{\alpha)}, \\
\Delta^{\mu \nu \alpha \beta \equiv} \equiv & \frac{1}{24} L(L-1)(L-2)(L-3) \widehat{K}^{\mu \nu \alpha \beta}+\frac{1}{6} L^{2}(L-1)(L-2) \widehat{K}^{(\mu \nu \alpha} \widehat{K}^{\beta)}+ \\
& +\frac{1}{6} L^{2}(L-1)(L-2) \widehat{K}^{(\beta} \widehat{K}^{\mu \nu \alpha)}+\frac{1}{4} L^{2}(L-1)^{2} \widehat{K}^{(\mu \nu} \widehat{K}^{\alpha \beta)}- \\
& -\frac{1}{2} L^{3}(L-1) \widehat{K}^{(\mu \nu} \widehat{K}^{\alpha} \widehat{K}^{\beta)}-\frac{1}{2} L^{3}(L-1) \widehat{K}^{(\alpha} \widehat{K}^{\mu \nu} \widehat{K}^{\beta)}- \\
& -\frac{1}{2} L^{3}(L-1) \widehat{K}^{(\alpha} \widehat{K}^{\beta} \widehat{K}^{\mu \nu)}+L^{4} \widehat{K}^{(\mu} \widehat{K}^{\nu} \widehat{K}^{\alpha} \widehat{K}^{\beta)},
\end{aligned}
$$

причем симметризация производится по всем греческим индексам и

$$
A^{\left(i_{1} i_{2} \ldots i_{n}\right)} \equiv \frac{1}{n !}\left(A^{i_{1} i_{2} \ldots i_{n}}+A^{i_{2} i_{1} \ldots i_{n}}+\cdots+A^{i_{n} i_{n-1} \ldots i_{1}}\right)
$$

(необходимость симметризации связана с наличием свертки с полностью симметричным тензором $\left.p_{\mu} \ldots p_{\alpha}\right)$.

Полученные коэффициенты удовлетворяют следующим тождествам, которые неоднократно использовалось при вычислении однопетлевых диаграмм:

$$
\begin{aligned}
& \Delta^{\mu \nu}+\frac{1}{2} L(L-1) \widehat{K}^{\mu \nu}+L \widehat{K}^{(\mu} \Delta^{\nu)}=0, \\
& \Delta^{\mu \nu \alpha}+\frac{1}{6} L(L-1)(L-2) \widehat{K}^{\mu \nu \alpha}+\frac{1}{2} L(L-1) \widehat{K}^{(\mu \nu} \Delta^{\alpha)}+L \widehat{K}^{(\mu} \Delta^{\nu \alpha)}=0, \\
& \Delta^{\mu \nu \alpha \beta}+\frac{1}{24} L(L-1)(L-2)(L-3) \widehat{K}^{\mu \nu \alpha \beta}+\frac{1}{6} L(L-1)(L-2) \widehat{K}^{(\mu \nu \alpha} \Delta^{\beta)}+ \\
& \quad+\frac{1}{2} L(L-1) \widehat{K}^{(\mu \nu} \Delta^{\alpha \beta)}+L \widehat{K}^{(\mu} \Delta^{\nu \alpha \beta)}=0
\end{aligned}
$$


и т.д., а также аналогичным тождествам, которые получаются, если $K$ и $\Delta$ поменять местами.

Приведем теперь наиболее простой пример применения тождеств (П.5) и (П.7). Рассмотрим величину

$$
\left\langle(L-3)(N n)^{\alpha}(K n)^{-1}\right\rangle
$$

где $N^{\mu \nu \ldots \alpha}$ - полностью симметричный тензор с $L-3$ индексами (аналогичные выражения часто встречаются при вычислении фейнмановских диаграмм). Записывая $(L-3)(N n)^{\alpha}$ в виде $\delta^{\alpha}(N n)$ и используя правило $(\Pi .5)$, получаем следуюшее соотношение:

$$
\left\langle(L-3)(N n)^{\alpha}(K n)^{-1}\right\rangle=-\left\langle(N n) \delta^{\alpha}(K n)^{-1}\right\rangle .
$$

Вспоминая выражение (П.10) для $\Delta^{\alpha}$, мы немедленно приходим к тождеству

$$
\left\langle(L-3) \widehat{N}^{\alpha}\right\rangle=L\left\langle\widehat{N} \widehat{K}^{\alpha}\right\rangle .
$$

Рассмотрение более сложных случаев производится совершенно аналогично, хотя далеко не всегда является очевидной связь между различными выражениями, получаемая подобным образом.

\section{Список литературы}

[1] R. Jackiw. Phys.Rev. 1974. V. D9. P. 1686.

[2] J.S. Schwinger. Phys. Rev. 1951. V. 82. P. 664.

[3] Б. С. де Bumm. Динамическая теория групп и полей. М.: Наука, 1987.

[4] A. O. Barvinsky, G. A. Vilkovisky. Phys.Rep. 1985. V. 119. P. 1.

[5] G. t'Hooft, M. Veltman. Ann. Inst. Henri Poincaré. 1974. V. 20. P. 69.

[6] P. Pronin, K. Stepanyantz. Gravity, Particles and Space-Time. Eds P. Pronin and G. Sardanasvili. Singapure: World Scientific, 1996. P. 441; Grav. and Cosmology. 1996. V. 2. P. 38 .

[7] П.И. Пронин, К. В. Степаньяни. ТМФ. 1996. Т. 109. № 2. С. 215; P.Pronin, K. Stepanyantz. Hep-th 9604038.

[8] P.I. Pronin, K. V. Stepanyantz. Hep-th 9605206.

[9] P.I. Pronin, K. V. Stepanyantz. In: New Computing Technick in Physics Research. IV. Eds B. Denby and D. Perred-Gallix. Singapure: World Scientific, 1995. P. 187.

[10] A. Hearn. REDUCE USER'S MANUAL, version 3.3. The Rand publication CP78 (Rev. 7.87), 1987.

[11] E.S. Fradkin, A.A. Tseytlin. Higher Derivative Quantum Gravity: One-loop Counterterms and Asymptotic Freedom: Preprint of P. Lebedev Physical Institute, № 170. Moscow, 1981; Phys.Lett. 1981. V. B104. P. 377; Nucl.Phys. 1982. V. B201. P. 469.

[12] R. E. Kallosh, O. V. Tarasov, I. V. Tyutin. Nucl.Phys. 1978. V. B137. P. 145.

[13] A. A. Slavnov. Nucl.Phys. 1971. V. B31. Р. 301; A. А. Славнов. ТМФ. 1972. Т. 13. С. 174

Поступила в редакцию 17.VII.1996 г.

\section{P. I. Pronin, K. V. Stepanyantz \\ ONE-LOOP DIVERGENCES FOR THEORIES WITH ARBITRARY NONMINIMAL OPERATOR IN THE CURVED SPACE}

We present a master formula for the divergent part of the one-loop effective action for an arbitrary nonminimal operator in the 4-dimensional curved space. 